\title{
Electroweak phase transition triggered by fermion sector
}

\author{
Qing-Hong Cao, ${ }^{a, b, c}$ Katsuya Hashino, ${ }^{b} \mathbf{X u}$-Xiang Li, ${ }^{a}$ Zhe $\operatorname{Ren}^{d, e}$ and \\ Jiang-Hao $\mathbf{Y} \mathbf{u}^{d, e, b, f, g}$ \\ ${ }^{a}$ Department of Physics and State Key Laboratory of Nuclear Physics and Technology, Peking \\ University, \\ Beijing 100871, China \\ ${ }^{b}$ Center for High Energy Physics, Peking University, \\ Beijing 100871, China \\ ${ }^{c}$ Collaborative Innovation Center of Quantum Matter, \\ Beijing 100871, China \\ ${ }^{d}$ CAS Key Laboratory of Theoretical Physics, Institute of Theoretical Physics, Chinese Academy \\ of Sciences, \\ Beijing 100190, P. R. China \\ e School of Physical Sciences, University of Chinese Academy of Sciences, \\ Beijing 100049, P.R. China \\ ${ }^{f}$ School of Fundamental Physics and Mathematical Sciences, Hangzhou Institute for Advanced \\ Study, University of Chinese Academy of Sciences, \\ Hangzhou 310024, China \\ ${ }^{g}$ International Center for Theoretical Physics Asia-Pacific, \\ Beijing/Hangzhou, China \\ E-mail: qinghongcao@pku.edu.cn, hashino@pku.edu.cn, \\ jukcoeng@pku.edu.cn, renzhe@itp.ac.cn, jhyu@itp.ac.cn
}

ABSTRACT: To realize first-order electroweak phase transition, it is necessary to generate a barrier in the thermal Higgs potential, which is usually triggered by scalar degree of freedom. We instead investigate phase transition patterns in pure fermion extensions of the standard model, and find that additional fermions with mass hierarchy and mixing could develop such a barrier and realize a strongly first-order phase transition in such models. In the Higgs potential with polynomial parametrization, the barrier can be generated in the following two patterns by fermionic reduction effects: (I) positive quadratic term, negative cubic term and positive quartic term or (II) positive quadratic term, negative quartic term and positive higher dimensional term, such as dimensional 6 operator.

Keywords: Beyond Standard Model, Effective Field Theories, Higgs Physics, Thermal Field Theory

ARXIV EPRINT: 2103.05688 


\section{Contents}

1 Introduction 1

2 Multi-scale effective potential $\quad 5$

3 Extended fermion model 10

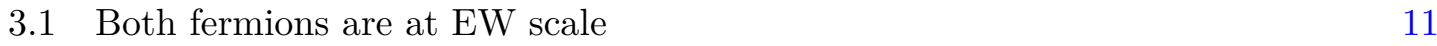

$\begin{array}{ll}3.2 & \text { Both fermions are at } \mathrm{TeV} \text { scale } \\ \end{array}$

3.2.1 $m_{L} \gg m_{N} \gg y_{N} v$ case $r 2$

3.2.2 $m_{L} \sim m_{N} \gg y_{N} v$ case 13

3.3 One fermion is at $\mathrm{TeV}$ scale and another is at $\mathrm{EW}$ scale 13

4 Numerical results on phase transition pattern $\quad 17$

$\begin{array}{llr}5 & \text { Summary } & 24\end{array}$

A Derivation of the effective potential in case $B$ and $C$ and normalized effective couplings

A.1 Case B-1: $m_{L} \gg m_{N} \gg y_{N} v \quad 25$

A.2 Case B-2: $m_{L} \sim m_{N} \gg y_{N} v \quad 27$

A.3 Case C: $m_{L} \gg m_{N} \sim y_{N} v$

A.4 Normalized effective couplings in the model with $y_{N}=3$ and 4 in case C $\quad 29$

$\begin{array}{ll}\text { B Vacuum stability and Landau pole } & 30\end{array}$

\section{Introduction}

Although the standard model (SM) like Higgs boson has been discovered at the large hadron collider [1, 2], currently the shape of the Higgs potential is still undetermined [3]. It is well known that dynamics of the electroweak phase transition (EWPT), governed by the shape of the Higgs potential, is quite important to describe the early universe at the electroweak scale. In particular, the first-order EWPT is very interesting, because it is one of the three necessary conditions in the electroweak baryogenesis scenario to explain baryon asymmetry of the universe [4], and furthermore, shape of the potential could be tested by the gravitational waves which are produced by the collision of bubbles via the first-order EWPT [5-11].

To realize first-order EWPT, it is necessary to develop a sizable potential barrier in the thermal Higgs potential. In the SM, although there are thermal loop corrections from the 
gauge bosons, the Higgs boson mass is too large to realize the first-order phase transition. The thermal Higgs potential in the SM, parametrized by various polynomial terms, reads

$$
V_{\mathrm{eff}}^{S M}(\varphi, T) \simeq D\left(T^{2}-T_{0}^{2}\right) \varphi^{2}-E T \varphi^{3}+\frac{\lambda_{T}}{4} \varphi^{4},
$$

where $\varphi$ is the classical background in the SM Higgs field and $D, T_{0}$ and $E$ are parameters independent of temperature $T$ and $\lambda_{T}$ depends on $T$ through logarithmic term. The coefficients $D T_{0}^{2}$ and $\lambda_{T}$ have dominant contributions from the tree-level potential, while $E$ can only be generated from the loop effect of gauge bosons:

$$
E=\frac{1}{4 \pi v^{3}}\left(2 m_{W}^{3}+m_{Z}^{3}\right) \simeq 10^{-2} .
$$

This term $E$ has much smaller contribution on the potential than other terms at $T \sim \varphi$, and thus the cubic term could be ignored. In such a case, the potential only has quadratic and quartic term of $\varphi$ :

$$
V_{\mathrm{eff}}^{S M}(\varphi, T) \simeq D\left(T^{2}-T_{0}^{2}\right) \varphi^{2}+\frac{\lambda_{T}}{4} \varphi^{4} .
$$

Thus, the first-order EWPT cannot be realized in the SM. Indeed, the lattice simulation says that the EWPT in the SM is not first-order but crossover $[12,13]$. One needs to increase size of the cubic term in the potential to obtain first-order EWPT. Usually, the cubic effect can be enhanced by degrees of freedom of additional bosons, because the cubic term typically comes from thermal loop effects of bosons [14] and furthermore tree-level effects of scalar bosons [15]. Thus it motivates various bosonic extensions of the SM, such as extended scalar boson models, and extended gauge sectors. On the contrary, it is usually believed that the fermion could not contribute to the cubic term. This could be understood from the one-loop finite temperature bosonic and fermionic corrections on the Higgs potential in the high temperature limit:

$$
\Delta V_{T} \simeq \begin{cases}-\frac{\pi^{2}}{90} T^{4}+\frac{M^{2}(\varphi)}{24} T^{2}-\frac{M^{3}(\varphi)}{12 \pi} T-\frac{M^{4}(\varphi)}{64} \ln \left(\frac{M^{2}(\varphi)}{\alpha_{B} T^{2}}\right)+\ldots, & \text { boson } \\ \frac{7 \pi^{2}}{720} T^{4}-\frac{M^{2}(\varphi)}{48} T^{2}-\frac{M^{4}(\varphi)}{32} \ln \left(\frac{M^{2}(\varphi)}{\alpha_{F} T^{2}}\right)+\ldots, & \text { fermion }\end{cases}
$$

where the cubic term $M^{3}(\varphi)$ does not appear in the fermionic thermal potential.

In this work, we consider phase transition patterns in fermionic extensions of the SM, and investigate whether the first-order phase transition could be triggered by pure fermion effects. In this case, although the cubic term cannot be enhanced, it is still possible to realize first-order EWPT by decreasing the quadratic and quartic contributions or adding higher dimensional terms in the Higgs potential. If there is only one additional fermion added to the SM, it is very hard to satisfy such conditions in the Higgs potential. However, if there are several additional fermions contributed to the Higgs potential, the situation might be different. This motivates us to consider extended models with several fermions included, and the simplest one is adding two fermions in the SM. A phase transition with several fermions has been considered in refs. [16-22]: refs. [16-19] discussed the first-order EWPT in the model with new fermion and scalar boson or with three new fermions, which 
are EW scale. Refs. [20, 21] considered the possibility of first-order phase transition in the model with new fermions for $\mathrm{TeV}$ and EW scale, however, ref. [20] includes the TeV fermion in the effective potential at EW scale. Ref. [22] studied electroweak symmetry nonrestoration in the extended fermion model. On the other hand, we would like to explore various mass hierarchy regions and obtain the most generic phase transition patterns by utilizing the multi-scale effective Higgs potential in the effective field theory framework. In particular, we take an effective field theory approach to study the effective Higgs potential with matching and running procedure systematically with avoiding artificial large logarithm in the potential. With the complete loop and running effects encoded in the Higgs potential, we find not only the scenario considered in literature $[20,21]$ but also new scenario could realize first-order EWPT.

To be specific, we consider a simple extended fermion model with two new fermions, such as vector like leptons, one $\mathrm{SU}(2)$ doublet fermion and one neutral singlet fermion, and systematically discuss various possibilities of how to generate a sizable barrier in the potential. The mass hierarchy patterns of two new fermions can be divided into three categories as following:

(A) both fermions are at the EW scale. In this mass region only the EW scale physics is involved and there is no need to perform integrating-out and matching procedure. Therefore, to obtain a sizable barrier, it is necessary to decrease the size of quadratic and quartic terms in the potential eq. (1.1) and thus make the quadratic and quartic terms comparable to the small cubic term due to the SM gauge boson mentioned above. However, it is impossible to realize such small quadratic term because new fermion contribution to the quadratic term has the same sign with the SM contribution and cancellation cannot happen. Therefore in this case the quadratic term $\varphi^{2}$ cannot be decreased to the same size as the cubic term to generate a sizable barrier and thus additional degree of freedom of additional bosons or more complex fermion sector is required [16-19].

(B) both fermions are at the $\mathrm{TeV}$ scale. In order to analyze the potential at the EW scale, heavy fields with the $\mathrm{TeV}$ scale mass should be integrated out, otherwise large logarithmic terms could be developed in the potential and thus the perturbation expansion might become invalid. Ref. [20] considers a similar model with heavy fermions at $\mathrm{TeV}$ scale, however, they do not take into account such a treatment and thus the barrier might be appeared due to artificially large logarithm. In this work, we utilize a systematical method to get the effective potential with matching and running procedure [21]. In this case, integrating out heavy particles only contributes to higher dimensional operators and cannot give rise to large negative quartic coupling in the potential. Therefore although positive higher dimensional contributions are obtained by integrating out new fermions, there is no enough negative quartic contribution to develop a barrier.

(C) one fermion is at the TeV scale and another is at the EW scale. Similar to the case (B), one integrates out the TeV scale heavy fermion and obtain the higher dimension operators. However, different from the case (B), the EW scale new fermion cannot be integrated out, and thus the mixing effects between heavy and light fermions 
have new contribution on the potential at the EW scale. Typically the larger mixing effect causes the more negative quartic coupling in the Higgs potential. In this case there are both high dimensional operators from $\mathrm{TeV}$ scale fermion contribution and thermal effects from EW scale fermion contribution [21, 22], which add up to generate positive quadratic, negative quartic and high dimensional operator in the potential. It could develop a sizable barrier and a first-order EWPT is realized.

On the other hand, when the mixing effect is not so large, a new scenario appears to realize first-order phase transition. In this case, the quartic coupling is small but still positive, and mixing effects would not cause negative quartic coupling, and thus the quartic term is comparable to the cubic term in the SM. At the same time, the quadratic term receives a positive contribution from integrating out heavy fermion, which is opposite to the SM tree-level contribution. Thus, there are cancellation between the positive new contribution and the SM one, and thus the quadratic term is decreased to smaller size which is comparable to the cubic term in the SM. In total, the effective potential with comparable positive quadratic, negative cubic and positive quartic terms could realize firstorder EWPT.

Among the above cases, only the case (C) could realize first-order EWPT, and as discussed above a sizable barrier could be developed under the following two scenarios:

- (I) First scenario: positive $\varphi^{2}$ term, negative $\varphi^{3}$ term and positive $\varphi^{4}$ term

$$
V_{\text {eff }}(\varphi, T) \simeq \frac{1}{2} \mu^{2} \varphi^{2}-\frac{1}{3} \lambda_{3} \varphi^{3}+\frac{1}{4} \lambda \varphi^{4} \quad\left(\mu^{2}, \lambda_{3}, \lambda>0\right),
$$

where negative cubic term only comes from the SM as shown eq. (1.2). The potential of this scenario looks like one of the SM in eq. (1.1), however, the fermion model has additional reductions in $\varphi^{2}$ and $\varphi^{4}$ terms through new fermion effects. Then a barrier can be generated in the effective potential, because the quadratic and quartic terms can be almost same size as cubic term.

- (II) Second scenario: positive $\varphi^{2}$ term, negative $\varphi^{4}$ term and positive high dimensional term of heavy fermion effect

$$
V_{\mathrm{eff}}(\varphi, T) \simeq \frac{1}{2} \mu^{2} \varphi^{2}-\frac{1}{4} \lambda \varphi^{4}+\frac{1}{6} \gamma \varphi^{6} \quad\left(\mu^{2}, \lambda, \gamma>0\right) .
$$

This scenario has larger fermion effects than scenario (I), and then the quartic term becomes negative. Thus a large barrier can be generated in the potential without cubic term in scenario (II).

In the following, we investigate the multi-scale effective potential using the matching and running procedure in section 2. The detail of extended fermion model is shown in section 3 and we check the temperature dependence of coefficients of $\varphi^{n}$ in order to discuss the detail of generation of a barrier in the model. In section 4 , we explore the parameter region where a barrier appears in the potential. The conclusion is drawn in section 5 . 


\section{Multi-scale effective potential}

Here we consider a general ultraviolet theory containing several fields: the SM particles, new particles at the EW scale and new heavy particles at the TeV scale. According to ColemanWeinberg's treatment [23-26], the one-loop effective potential develops the logarithmic term $\log \frac{m^{2}}{Q^{2}}$ where $m^{2}$ is the mass-squared running over all the relevant particles, and $Q$ is the renormalization scale taken to be the EW scale in the effective potential. Since there are new heavy particles, the effective potential would contain at least the large logarithmic term $\log \frac{M_{\text {heavy }}^{2}}{Q^{2}}$, in which $M_{\text {heavy }}$ is the mass of heavy particles and $Q$ is the renormalization scale. In order to analyse the EWPT, we take $Q$ in the effective potential as EW scale. Since there are new heavy particles at EW scale, the effective potential would contain at least the large logarithmic term $\log$ heavy $\log \frac{M_{\text {heavy }}^{2}}{Q^{2}}$ in which $M_{\text {heavy }}$ is the mass of heavy particles. To solve this problem, several kinds of methods, such as the renormalization group (RG) improved effective potential [23-26], the matching and running method [27-44], are used to get the effective potential at the EW scale. In this work, we utilize the matching and running method in ref. [44] and generalize the formalism to cases with several new particles. In the matching and running method, we perform both the matching between effective potential and the effective potential without heavy $\mathrm{TeV}$ particle at the $\mathrm{TeV}$ scale, and then do RG running down to the effective potential at the EW scale. The following matching and running procedure is used to obtain the effective potential at the EW scale:

1. Define the ultraviolet (UV) Lagrangian containing all fields,

2. Calculate the one-loop effective potential at high energy (HE) scale,

3. Absorb the divergence in the calculation by the renormalization and tadpole scheme,

4. Match the potential at the HE scale with the one containing light fields and high dimensional operators at low energy (LE) scale at matching scale $Q_{M}$ by the matching condition,

5. Evaluate RG runnings of parameters in the effective potential from the matching scale $Q_{M}$ to the EW scale $Q_{E W}$.

6. Obtain the effective potential at the EW scale.

This procedure on calculation of the effective potential at the EW scale is summarized in figure 1. The general UV Lagrangian containing massive bosonic and fermionic degree of freedom at the HE scale could be written as

$$
\begin{aligned}
\mathcal{L}= & \frac{1}{2}\left|D_{\mu} \phi_{(i)}\right|^{2}+\frac{1}{2}\left|D_{\mu} \Phi_{(i)}\right|^{2}+i \bar{f}_{(i)} \not D f_{(i)}+i \bar{F}_{(i)} \not D F_{(i)} \\
& -\lambda_{(i, j, k, l),\{m, n, o, p\}}^{(a, b, c, d),\{e, f, g, h\}} \phi_{(i)}^{m} \phi_{(j)}^{n} \phi_{(k)}^{o} \phi_{(l)}^{p} \Phi_{(a)}^{e} \Phi_{(b)}^{f} \Phi_{(c)}^{g} \Phi_{(d)}^{h} \\
& -Y_{(i, j, k),\{m, n, o\}}^{(a, b, c),\{g, h, i\}} \phi_{(i)}^{m} \Phi_{(a)}^{g} \bar{f}_{(j)}^{n} f_{(k)}^{o} \bar{F}_{(b)}^{h} F_{(c)}^{i},
\end{aligned}
$$




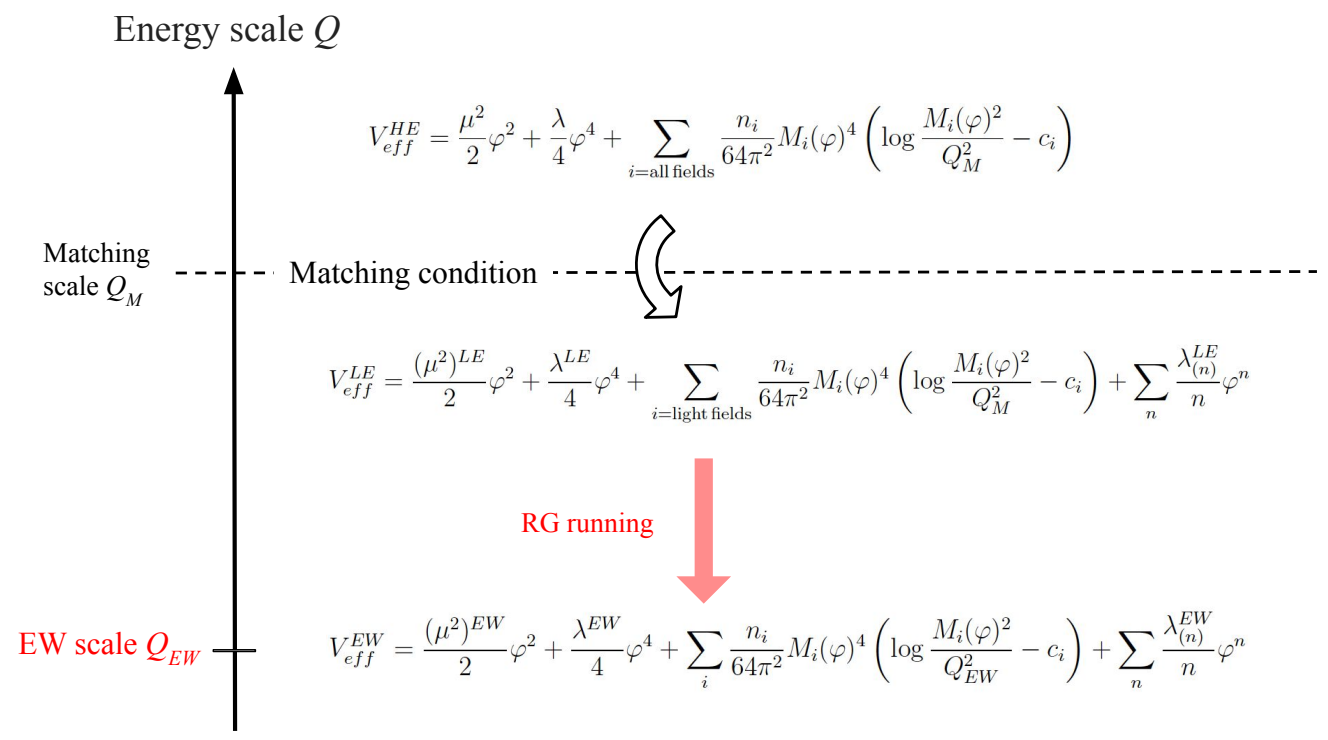

Figure 1. The procedure of obtaining the effective potential at the EW scale. The effective potential contains the classical background $\varphi$ of the SM Higgs field and the divergence in it is absorbed by the $\overline{\mathrm{MS}}$ scheme. At the HE scale, the effective potential $V_{\mathrm{eff}}^{H E}$, which is calculated at the matching scale $Q_{M}$, contains all fields. At the LE scale, the effective potential $V_{\text {eff }}^{L E}$ containing light fields and high dimensional operators of heavy fields can be obtained by the matching condition eq. (2.15). After performing the RG running from $Q_{M}$ to $Q_{E W}$, we obtain the effective potential at the EW scale $V_{\mathrm{eff}}^{E W}$.

where $\phi_{(i)}^{m}\left(f_{(i)}\right)$ and $\Phi_{(i)}\left(F_{(i)}\right)$ are light and heavy boson (fermion) fields, superscript like $m$ is the power of field and subscript like $(i)$ is the kind of field. For simplicity, kinetic mixing term is not included in the Lagrangian and we assume only the SM-like Higgs boson has the vacuum expectation value.

First let us consider the HE effective potential at the TeV scale. The effective potential can be obtained by the functional trace of action regarding the Lagrangian, such as $\log \operatorname{det}\left(\frac{\delta^{2} \mathcal{L}}{\delta \phi_{(i)} \delta \phi_{(j)}}\right)[45]$.

$$
V^{H E}=\frac{\mu^{2}}{2} \varphi^{2}+\frac{\lambda}{4} \varphi^{4}+V_{C W}^{\text {all }}(Q)+\frac{\delta \mu^{2}}{2} \varphi^{2}+\frac{\delta \lambda}{4} \varphi^{4}+V_{d i v}^{H E},
$$

where $V_{C W}^{a l l}(Q)$ is Coleman and Weinberg part of the effective potential:

$$
V_{C W}^{a l l}(Q)=\sum_{i=\text { all fields }} \frac{n_{i}}{64 \pi^{2}} M_{i}(\varphi)^{4}\left(\log \frac{M_{i}(\varphi)^{2}}{Q^{2}}-c_{i}\right),
$$

where $n_{i}$ is degree of freedom of the $i$-th field, $M_{i}(\varphi)^{2}$ is its field dependent mass, $c_{i}=\frac{3}{2}$ (for scalar bosons and fermions), $\frac{5}{6}$ (for gauge bosons). The $\delta \mu^{2}$ and $\delta \lambda$ in eq. (2.2) are respectively the counter terms of the quadratic and quartic $\varphi$ terms and

$$
V_{d i v}^{H E}=\frac{Q^{2 \epsilon}}{\bar{\epsilon}} \sum_{i} \frac{n_{i}}{64 \pi^{2}}\left(M_{i}(\varphi)^{2}\right)^{2-\epsilon}
$$


where $\frac{1}{\bar{\epsilon}}=\frac{1}{\epsilon}-\gamma+\log 4 \pi$. Then the $\overline{\mathrm{MS}}$ scheme is utilized to absorb the UV divergence as follows

$$
\left.\frac{\partial^{2}}{\partial \varphi^{2}}\left(V_{d i v}^{H E}+\frac{\delta \mu^{2}}{2} \varphi^{2}+\frac{\delta \lambda}{4} \varphi^{4}\right)\right|_{\varphi=0}=0,\left.\quad \frac{\partial^{4}}{\partial \varphi^{4}}\left(V_{d i v}^{H E}+\frac{\delta \mu^{2}}{2} \varphi^{2}+\frac{\delta \lambda}{4} \varphi^{4}\right)\right|_{\varphi=0}=0
$$

The effective potential under the $\overline{\mathrm{MS}}$ scheme is

$$
V_{\mathrm{eff}}^{\overline{\mathrm{MS}}}=\frac{\mu^{2}}{2} \varphi^{2}+\frac{\lambda}{4} \varphi^{4}+V_{C W}^{\text {all }}(Q) .
$$

In order to get the HE effective potential, one also need to fix the tadpole schemes [4652]. In the tadpole schemes, the shift of scalar field with $v_{0}\left(\varphi \rightarrow \varphi+v_{0}\right)$ shows up, where the subscript represents the bare parameter. This shift evolves a linear term in the effective potential: $-\left(\mu_{0}^{2}+\lambda_{0} v_{0}^{2}\right) v_{0} \varphi$, and the form of effective potential containing tree-level and counter term is

$$
\begin{aligned}
V_{e f f, 0, c}^{T a d}= & v\left(\mu^{2}+\lambda v^{2}\right) \varphi+\frac{\mu^{2}+3 \lambda v^{2}}{2} \varphi^{2}+\lambda v \varphi^{3}+\frac{\lambda}{4} \varphi^{4}+\left(v \delta \mu^{2}+\delta \lambda v^{3}+\delta v\left(\mu^{2}+3 \lambda v^{2}\right)\right) \varphi \\
& +\frac{\delta \mu^{2}+3 v(\delta \lambda v+2 \lambda \delta v)}{2} \varphi^{2}+(\delta \lambda v+\lambda \delta v) \varphi^{3}+\frac{\delta \lambda}{4} \varphi^{4}+O\left(\lambda^{3}\right) .
\end{aligned}
$$

The tadpole condition for fixing $v$ and $\delta v$ is

$$
\langle 0|\varphi| 0\rangle=0 \Rightarrow-i \delta t+i \Gamma_{\varphi}^{(1)}=0
$$

where $\delta t$ appears in the effective potential as the linear term of $\varphi, V_{e f f, 0}^{\text {Tad }} \supset \delta t \varphi$, and $\Gamma_{\varphi}$ is the 1-point function of $\varphi$. The tadpole conditions at the tree-level and the one-loop level are

$$
v=\sqrt{-\frac{\mu^{2}}{\lambda}}, \quad-i\left(v \delta \mu^{2}+\delta \lambda v^{3}+\delta v\left(\mu^{2}+3 \lambda v^{2}\right)\right)+i \Gamma_{\varphi}^{(1), 1-l o o p}=0 .
$$

Other counter terms $\delta \mu^{2}, \delta \lambda$ are fixed by 2-point and 4-point function of $\varphi$. If the divergence parts are absorbed by the counter terms, such as the $\overline{\mathrm{MS}}$ scheme, the renormalization conditions are

$$
-i\left(\delta \mu^{2}+3 v\left(\delta \lambda v+2 \lambda \delta v^{d i v}\right)\right)+i \Gamma_{\varphi}^{(2), d i v}=0, \quad-i 6 \delta \lambda+i \Gamma_{\varphi}^{(4), d i v}=0
$$

This tadpole scheme is called Fleischer-Jegerlehner tadpole scheme (FJTS). Note that $\delta v$ contains finite term while $\delta \mu^{2}$ and $\delta \lambda$ have terms with $1 / \bar{\epsilon}$. Then the HE effective potential is

$$
\begin{aligned}
V_{\mathrm{eff}}^{F J T S}= & \frac{\mu^{2}}{2} \varphi^{2}+\frac{\lambda}{4} \varphi^{4}+V_{C W}^{\text {all }}(Q) \\
& +\left[\frac{\delta \mu^{2}}{2} \varphi^{2}+\frac{\delta \lambda}{4} \varphi^{4}+\sum_{i} \frac{n_{i}}{64 \pi^{2}} \frac{M_{i}^{4}(\varphi)}{\bar{\epsilon}}\right]
\end{aligned}
$$


For spontaneously symmetry breaking $\lambda \phi^{4}$ theory

$$
\mathcal{L}=\frac{1}{2}\left(\partial_{\mu} \varphi\right)^{2}-\frac{1}{2} \mu^{2} \varphi^{2}-\frac{1}{4} \lambda \varphi^{4}, \quad \mu^{2}<0, \lambda>0
$$

all divergences in the effective potential cancel with each other and $V_{\mathrm{eff}}^{F J S}=V_{\mathrm{eff}}^{\overline{\mathrm{MS}}}$. In the following analysis, we use the effective potential with the $\overline{\mathrm{MS}}$ scheme.

After obtaining the HE effective potential, we start to consider the matching between the HE and LE effective potentials. The HE effective potential is given in eq. (2.11), while the LE effective potential at the same UV scale can be written similarly with less field degree of freedom. Therefore, the HE and LE effective potential at the matching scale $Q_{M}$ are

$$
\begin{aligned}
V_{\mathrm{eff}}^{H E} & =\frac{\mu^{2}}{2} \varphi^{2}+\frac{\lambda}{4} \varphi^{4}+V_{C W}^{\text {all }}\left(Q_{M}\right), \\
V_{\mathrm{eff}}^{L E} & =\frac{\left(\mu^{2}\right)^{L E}}{2} \varphi^{2}+\frac{\lambda^{L E}}{4} \varphi^{4}+V_{C W}^{\text {light }}\left(Q_{M}\right)+\sum_{n \geq 6} \frac{\lambda_{(n)}^{L E}}{n} \varphi^{n},
\end{aligned}
$$

where $V_{C W}^{\text {light }}$ is a part of $V_{C W}$ with respect to only the light fields. The coefficient of $\varphi^{n}$ due to heavy fields, $\lambda_{(n)}$, is fixed by the matching conditions at the matching scale $Q_{M}$ :

$$
\begin{aligned}
\left(\mu^{2}\right)^{L E} & =\left(1+2 \delta Z_{\varphi}^{\text {heavy }}\right) \mu^{2}+\left.\frac{\partial^{2}}{\partial \varphi^{2}} V_{C W}^{\text {heavy }}\left(Q_{M}\right)\right|_{\varphi=0}, \\
\lambda^{L E} & =\left(1+4 \delta Z_{\varphi}^{\text {heavy }}\right) \lambda+\left.\frac{1}{6} \frac{\partial^{4}}{\partial \varphi^{4}} V_{C W}^{\text {heavy }}\left(Q_{M}\right)\right|_{\varphi=0}, \\
\lambda_{(n)}^{L E} & =\left.\left(1+n \delta Z_{\varphi}\right) \frac{\partial^{n}}{\partial \varphi^{n}} V_{C W}^{\text {heavy }}\left(Q_{M}\right) \simeq \frac{1}{(n-1) !} \frac{\partial^{n}}{\partial \varphi^{n}} V_{C W}^{\text {heavy }}\left(Q_{M}\right)\right|_{\varphi=0},
\end{aligned}
$$

where $V_{C W}^{\text {heavy }}$ is a part of $V_{C W}$ with respect to heavy fields. Here, differential terms on the Coleman-Weinberg potential $\partial^{n} V_{C W}^{\text {heavy }} / \partial \varphi^{n}$ in these eqs. (2.15), (2.16) and (2.17) represent threshold effects from integrating out the heavy fields. $\delta Z_{\varphi}^{\text {heavy }}$ denotes the wave function correction at the one-loop level according to the redefined $\varphi$ in LE scale after matching.

Since the contributions from light fields are the same between HE and LE theory, the corrections from light fields $\delta Z_{\varphi}^{\text {light }}$ do not appear in the right hand side of eqs. (2.15) and (2.16). In eqs. (2.15) and (2.16), although the $\delta Z_{\varphi}^{\text {heavy }}$ is the same order as the ColemanWeinberg corrections, since $\left(\mu^{2}\right)^{L E}$ and $\lambda^{L E}$ at the EW scale can always be determined by the stationary and mass conditions, such as eq. (2.20), the radiative corrections, including the $\delta Z_{\varphi}^{\text {heavy }}$, are not relevant in our study. Finally, in eq. (2.17), the $\delta Z_{\varphi}$ term is negligible in the right hand side because its contribution belongs to a next-to-next-leading order contribution to the $\lambda_{(n)}^{L E}$.

After obtaining the LE effective potential at the matching scale $Q_{M}$, we could obtain the effective potential at the EW scale $Q_{E W}$ via the RG running. The beta function can be obtained through the RG equation of the effective potential at the LE scale:

$$
\sum_{a}\left(\beta_{a} \frac{\partial}{\partial \lambda_{a}}-\gamma_{\varphi} \varphi \frac{\partial}{\partial \varphi}-\frac{\partial}{\partial Q}\right) V_{\mathrm{eff}}^{L E}=0
$$


By using the beta functions, the effective potential at the EW scale is given by

$$
V_{\mathrm{eff}}^{E W}=\frac{\left(\mu^{2}\right)^{E W}}{2} \varphi^{2}+\frac{\lambda^{E W}}{4} \varphi^{4}+V_{C W}^{l i g h t}\left(Q_{E W}\right)+\sum_{n} \frac{\lambda_{(n)}^{E W}}{n} \varphi^{n},
$$

where $\left(\mu^{2}\right)^{E W}, \lambda^{E W}$ and $\lambda_{(n)}^{E W}$ terms are parameters at the EW scale. Actually, the $\left(\mu^{2}\right)^{E W}$ and $\lambda^{E W}$ are determined not by eq. (2.15) and (2.16) with RG running, but by the stationary condition and second derivative of the potential at the EW scale:

$$
\left.\frac{\partial V_{\text {eff }}}{\partial \varphi}\right|_{\varphi=v}=0,\left.\quad \frac{\partial^{2} V_{\text {eff }}}{\partial \varphi^{2}}\right|_{\varphi=v}=m_{h}^{2}
$$

which will replace the $\left(\mu^{2}\right)^{E W}$ and $\lambda^{E W}$ to quantities at EW scale like vacuum expectation value, the Higgs boson mass, $\lambda_{(n)}^{E W}$ and light fermion effects. Therefore, the radiative corrections to $\left(\mu^{2}\right)^{L E}$ and $\lambda^{L E}$ in eqs. (2.15) and (2.16) are not relevant in the potential at the EW scale.

The above effective potential at the EW scale only contains the zero temperature effects. The finite temperature effects $\Delta V_{T}$ and $V_{T}^{\text {ring }}$ should be added to the effective potential, in order to describe the phase transition. The 1-loop finite temperature effect $\Delta V_{T}$ can be written as

$$
\begin{aligned}
\Delta V_{T}= & \frac{T^{4}}{2 \pi^{2}}\left\{\sum_{i=\text { bosons }} n_{i} \int_{0}^{\infty} d x x^{2} \ln \left[1-\exp \left(-\sqrt{x^{2}+\left(M_{i}(\varphi) / T\right)^{2}}\right)\right]\right. \\
& \left.+\sum_{i=\text { fermions }} n_{i} \int_{0}^{\infty} d x x^{2} \ln \left[1+\exp \left(-\sqrt{x^{2}+\left(M_{i}(\varphi) / T\right)^{2}}\right)\right]\right\} .
\end{aligned}
$$

In high temperature limit, the finite temperature effects are given as following forms:

$$
\begin{aligned}
& \int_{0}^{\infty} d x x^{2} \ln \left[1-\exp \left(-\sqrt{x^{2}+a^{2}}\right)\right] \rightarrow-\frac{\pi^{4}}{45}+\frac{\pi^{2}}{12} a^{2}-\frac{\pi}{6}\left(a^{2}\right)^{3 / 2}-\frac{a^{4}}{32} \ln \left(\frac{a^{2}}{\alpha_{B}}\right)+\ldots, \\
& \int_{0}^{\infty} d x x^{2} \ln \left[1+\exp \left(-\sqrt{x^{2}+a^{2}}\right)\right] \rightarrow \frac{7 \pi^{4}}{360}-\frac{\pi^{2}}{24} a^{2}-\frac{a^{4}}{32} \ln \left(\frac{a^{2}}{\alpha_{F}}\right)+\ldots
\end{aligned}
$$

where $a=M(\varphi) / T, \log \alpha_{B}=2 \log 4 \pi-2 \gamma_{E}+3 / 2, \log \alpha_{F}=2 \log \pi-2 \gamma_{E}+3 / 2$ and $\gamma_{E}$ is Euler constant. One could also include the daisy ring effect with the resummation, $V_{T}^{\text {ring }}$, which is obtained to be

$$
V_{T}^{\text {ring }}=\frac{T}{12 \pi} \sum_{\text {i=bosons }} n_{i}\left(\left(M_{i}(\varphi)^{2}\right)^{3 / 2}-\left(M_{i}(\varphi, T)^{2}\right)^{3 / 2}\right), \quad M_{i}(\varphi, T)^{2}=M_{i}(\varphi)^{2}+\Pi_{i},
$$

where $\Pi_{i}$ is the thermal self-energy. Since we expect the self-energy effect to be smaller than other contributions, here we neglect this daisy resummation effect in the following discussions. According to refs. [54, 55], the additional contributions from the higher-dimensional operators in principle appear in the finite temperature part in eqs. (2.21) and (2.22). The 
thermal contributions can be obtained by one-loop calculations coming from diagrams including high dimensional operators. The diagrams are calculated by Feynman rules in the Lagrangian at LE scale with these operators. Such effects are in field dependent masses, such as equations in appendix A.2 of ref. [55]. We neglect these effects of high dimensional operators, which can be obtained by one-loop diagrams for heavy fields, in the field dependent masses, because these are typically two-loop level conditions to the effective potential.

In this section, the key result is the eq. (2.19), the effective potential at the EW scale, which serves as the starting point to describe how a sizable barrier can be generated in a model with heavy fields. ${ }^{1}$ In the following analysis, we will focus on the extended fermion model and explore the phase transition pattern in such models.

\section{$3 \quad$ Extended fermion model}

In this section, we apply the general treatment of the multi-scale effective potential to the extended fermion model, more specifically, the model with one isospin doublet and one neutral isospin singlet fermions which are vector like leptons. The Lagrangian with the doublet fermion $L^{T}=(N, E)$ and the neutral singlet fermions $N^{\prime}$ is

$$
-\mathcal{L}_{\text {fermion }} \supset y_{N}\left(\bar{L} \tilde{H} N^{\prime}+\text { h.c. }\right)+m_{L} \bar{L} L+m_{N} \bar{N}^{\prime} N^{\prime}
$$

where new model parameters are $y_{N}, m_{N}$ and $m_{L}$. In this Lagrangian, the mass matrix for the neutral fermion $N, N^{\prime}$ reads

$$
\mathcal{M}_{N}=\left(\begin{array}{cc}
m_{L} & \frac{\varphi}{\sqrt{2}} y_{N} \\
\frac{\varphi}{\sqrt{2}} y_{N} & m_{N}
\end{array}\right)
$$

After diagonalization, the field dependent masses for the mass eigenstates of the new fermions $N_{1}$ and $N_{2}$ are given by

$$
M_{N_{1}, N_{2}}^{2}(\varphi)=\frac{1}{2}\left(m_{L}^{2}+m_{N}^{2}+y_{N}^{2} \varphi^{2} \mp\left(m_{L}^{2}-m_{N}^{2}\right) \sqrt{1+\frac{2 y_{N}^{2} \varphi^{2}}{\left(m_{L}-m_{N}\right)^{2}}}\right) .
$$

In order to analyze behaviour of effective potential under different parameter region, we parameterize the potential into a following polynomial form:

$$
\begin{aligned}
V_{\mathrm{eff}}^{P}= & \lambda_{1, \text { eff }} \varphi+\frac{\mu_{\mathrm{eff}}^{2}}{2} \varphi^{2}+\frac{\lambda_{3, e f f}}{3} \varphi^{3}+\frac{\lambda_{\mathrm{eff}}}{4} \varphi^{4}+\frac{\lambda_{5, \text { eff }}}{5} \varphi^{5}+\frac{\gamma_{\mathrm{eff}}}{6} \varphi^{6}+\frac{\lambda_{7, e f f}}{7} \varphi^{7}+\frac{\delta_{\mathrm{eff}}}{8} \varphi^{8} \\
& +\frac{\lambda_{9, e f f}}{9} \varphi^{9}+\frac{\epsilon_{\mathrm{eff}}}{10} \varphi^{10}+\mathcal{O}\left(\varphi^{11}\right) .
\end{aligned}
$$

\footnotetext{
${ }^{1}$ In order to discuss in detail the phase transition, we will take into account the different multi-scale effective potential among other renormalization schemes, theoretical uncertainties $[56,57]$ and several ways to get the effective potential at the LE scale in future work [53].
} 
These coefficients, recognized as the effective couplings, are calculated by differentials of the effective potential with the finite temperature effect:

$$
\begin{aligned}
\mu_{\mathrm{eff}}^{2} & \left.\equiv \frac{\partial^{2}}{\partial \varphi^{2}}\left(V_{\mathrm{eff}}+\Delta V_{T}\right)\right|_{\varphi=0},\left.\quad \lambda_{\mathrm{eff}} \equiv \frac{1}{3 !} \frac{\partial^{4}}{\partial \varphi^{4}}\left(V_{\mathrm{eff}}+\Delta V_{T}\right)\right|_{\varphi=0}, \ldots \\
\lambda_{n, \text { eff }} & \left.\equiv \frac{1}{(n-1) !} \frac{\partial^{n}}{\partial \varphi^{n}}\left(V_{\mathrm{eff}}+\Delta V_{T}\right)\right|_{\varphi=0} .
\end{aligned}
$$

Among them the coefficient of the $\varphi^{4}$ term $\left(\lambda_{\text {eff }}\right)$ is important to generate a barrier in the following two scenarios: in scenario (I) where positive $\mu_{\text {eff }}^{2}$, negative $\lambda_{3, \text { eff }}$ and positive $\lambda_{\text {eff }}$ terms, a barrier may show up when the positive $\lambda_{\text {eff }}$ is closed to $E$ in eq. (1.2) by cancellation between the SM effect and the new fermion effect. On the other hand, a barrier may also be generated by scenario (II) where negative $\lambda_{\text {eff }}$ term has larger negative fermion effect than scenario (I). Before discussing the $T$ dependence of the effective couplings, we first estimate behaviour of $\lambda_{\text {eff }}^{T=0}$ both analytically and numerically. Such an effective coupling $\lambda_{\text {eff }}^{T=0}$ is roughly given by

$$
\lambda_{\text {eff }}^{T=0} \sim \lambda_{\text {eff }}^{S M}-2 \lambda_{(6)} v^{2}+\left.\frac{1}{6} \frac{\partial^{4} V_{1-l o o p}^{V L L}}{\partial \varphi^{4}}\right|_{\varphi=0}, \quad V_{1-l o o p}^{V L L}=\sum_{V L L} \frac{-4}{64 \pi^{2}} M_{i}(\varphi)^{4}\left(\log \frac{M_{i}(\varphi)^{2}}{v^{2}}-\frac{3}{2}\right),
$$

where $\lambda_{\text {eff }}^{S M}$ is the SM contribution containing the loop effects. Under finite temperature, we use the high temperature approximation for the SM effects in order to avoid the divergence of logarithmic term in the SM effects at $\varphi=0$. We note that this approximation becomes invalid at low temperature $m / T \sim 1$.

In the following, we will discuss the effective couplings in the potential in three mass parameter cases (A) $m_{L} \sim m_{N} \sim y_{N} v$, (B) $m_{L} \gg m_{N} \gg y_{N} v, m_{L} \sim m_{N} \gg y_{N} v$ and (C) $m_{L} \gg m_{N} \sim y_{N} v$. In some cases, mass parameters are chosen to be at the TeV scale, which could come from physics at even higher energy scale, such as new symmetry breaking, composite Higgs model and so on. But in this work we do not consider such UV completion above the $\mathrm{TeV}$ scale and only introduce the heavy mass parameter by hand.

\subsection{Both fermions are at EW scale}

In the case that both new fermions are at the EW scale $m_{L} \sim m_{N} \sim y_{N} v$, the effective potential does not encounter the multi-scale problem and thus there is no need to introduce high dimensional operators into the effective potential, and the field dependent masses for new fermions in eq. (3.3) are approximated as

$$
M_{N_{1}, N_{2}}^{2}(\varphi) \sim m_{L}^{2}+\frac{y_{N}^{2} \varphi^{2}}{2} \mp \sqrt{2} m_{L} y_{N} \varphi
$$

The effective potential at the EW scale is

$$
V_{\mathrm{eff}}^{E W}=\frac{\mu^{2}}{2} \varphi^{2}+\frac{\lambda}{4} \varphi^{4}+\sum_{i=N_{1}, N_{2}, S M} \frac{n_{i}}{64 \pi^{2}} M_{i}(\varphi)^{4}\left(\log \frac{M_{i}(\varphi)^{2}}{v^{2}}-c_{i}\right)
$$


where $n_{t}=-12, n_{W}=6, n_{Z}=3, n_{h}=1, n_{N G B}=3, n_{N_{1}, N_{2}}=-4$. The quartic coupling from new fermion effects is

$$
\lambda_{\text {eff }}^{T=0} \sim \lambda_{\text {eff }}^{S M}-\frac{y_{N}^{4}}{8 \pi^{2}}\left(\log \frac{m_{L}^{2}}{v^{2}}+\frac{8}{3}\right),
$$

where the second term may reduce the quartic coupling to the same level with $\lambda_{3, e f f}$.

Temperature dependence. From eqs. (2.19), (2.21) and (3.5), the quadratic and quartic terms originated from new fermions, including the $T$ dependence, are

$$
\begin{aligned}
\left(\mu_{\mathrm{eff}}^{\text {new fermions }}\right)^{2} & \simeq \frac{y_{N}^{2}}{12}\left(2 T^{2}-\frac{9 m_{L}^{2}}{\pi^{2}}\left(\ln \frac{\alpha_{F} T^{2}}{v^{2}}-\frac{3}{2}\right)\right), \\
\lambda_{\text {eff }}^{\text {new fermions }} & \simeq-\frac{y_{N}^{4}}{8 \pi^{2}}\left(\ln \frac{\alpha_{F} T^{2}}{v^{2}}-\frac{3}{2}\right)
\end{aligned}
$$

where the high temperature approximation is taken. In eq. (3.10), the first term proportional to the $T^{2}$ is dominant at high temperature while the second term with thermal logarithm is dominant at the temperature $T<m_{L}$. In total, the quadratic term from new fermion contribution is negative around the electroweak phase transition temperature. The new quadratic term has the same sign as the tree-level $\mu^{2}$ term in the effective potential, and cancellation between two terms cannot happen to obtain smaller overall quadratic term which is comparable to the cubic term from the SM gauge bosons. Therefore in this case there is no comparable contributions of positive quadratic, negative cubic and positive quartic terms in the potential to generate a sizable barrier. This is the reason why the first-order EWPT can not be realized in the simple fermion model [16-19].

\subsection{Both fermions are at $\mathrm{TeV}$ scale}

Since both fermions are at the $\mathrm{TeV}$ scale, the effective potential at the $\mathrm{EW}$ scale would develop large logarithmic term, and thus we use the matching-running method in section 2 to avoid such term. There are two parameter regions to be considered: $m_{L} \gg m_{N} \gg y_{N} v$ or $m_{L} \sim m_{N} \gg y_{N} v$. The detail of treatment of the multi-scale effective potential in case (B) is given in appendix A, and here we only list the main results.

\subsection{1 $m_{L} \gg m_{N} \gg y_{N} v$ case}

In this case, we consider a two-step matching and running because there are two heavy scales: very HE scale $m_{L}$ and HE scale $m_{N}$. We will consider the high dimensional operators up to dimensional 10 in this work. After the matching and running procedure, the effective potential at the EW scale is

$$
\begin{aligned}
V_{\mathrm{eff}}^{E W}= & \frac{\mu^{2}}{2} \varphi^{2}+\frac{\lambda}{4} \varphi^{4}+\sum_{i=S M} \frac{n_{i}}{64 \pi^{2}} M_{i}(\varphi)^{4}\left(\log \frac{M_{i}(\varphi)^{2}}{v^{2}}-c_{i}\right) \\
& +\frac{\lambda_{(6)}}{6} \varphi^{6}+\frac{\lambda_{(8)}}{8} \varphi^{8}+\frac{\lambda_{(10)}}{10} \varphi^{10}
\end{aligned}
$$


where $\lambda_{(n)}$ is the $n$-point coupling at the EW scale:

$$
\lambda_{(6)}=\gamma^{L E}-\beta_{6 \varphi}^{L E} \log \frac{m_{N}}{v}, \quad \lambda_{(8)}=\delta^{L E}-\beta_{8 \varphi}^{L E} \log \frac{m_{N}}{v}, \quad \lambda_{(10)}=\epsilon^{L E}-\beta_{10 \varphi}^{L E} \log \frac{m_{N}}{v} .
$$

The first and second terms in the right hand side are respectively the threshold effect in eq. (A.9) and the RG running term in eq. (A.10). If we exchange $m_{N}$ and $m_{L}$, the above calculations correspond to the case of $m_{N} \gg m_{L} \gg y_{N} v$.

\subsection{2 $m_{L} \sim m_{N} \gg y_{N} v$ case}

In this case, the field dependent masses of the new fermions are eq. (3.7). The form of the effective potential at the EW scale is the same as eq. (3.12) and dimensional 6, 8 and 10 operators at the EW scale are roughly

$$
\begin{aligned}
\lambda_{(6)} & \simeq \frac{y_{N}^{6}}{160 \pi^{2} m_{L}^{2}}\left(1+\frac{3}{8 \pi^{2}}\left(-3 y_{t}^{2}+\frac{3}{4} g_{1}^{2}+\frac{9}{4} g_{2}^{2}\right) \log \frac{m_{L}}{v}\right), \\
\lambda_{(8)} & \simeq \frac{y_{N}^{8}}{2240 \pi^{2} m_{L}^{4}}\left(1+\frac{1}{2 \pi^{2}}\left(-3 y_{t}^{2}+\frac{3}{4} g_{1}^{2}+\frac{9}{4} g_{2}^{2}\right) \log \frac{m_{L}}{v}\right), \\
\lambda_{(10)} & \simeq \frac{y_{N}^{10}}{16128 \pi^{2} m_{L}^{6}}\left(1+\frac{5}{8 \pi^{2}}\left(-3 y_{t}^{2}+\frac{3}{4} g_{1}^{2}+\frac{9}{4} g_{2}^{2}\right) \log \frac{m_{L}}{v}\right),
\end{aligned}
$$

where $y_{t}$ is the top Yukawa coupling and $g_{1}$ and $g_{2}$ are the gauge couplings of $\mathrm{U}(1)_{Y}$ and $\mathrm{SU}(2)_{L}$ symmetry, respectively.

The form of $\lambda_{(n)}$ differs between cases (B-1) and (B-2). For example, the dimensional 6 operators are $\lambda_{(6)}^{(\mathrm{B}-1)} \sim \frac{y_{N}^{6}}{16 \pi^{2} m_{L}^{2}}$ and $\lambda_{(6)}^{(\mathrm{B}-2)} \sim \frac{y_{N}^{6}}{160 \pi^{2} m_{L}^{2}}$. Here the $1 / 10$ factor difference comes from different effective interactions between $\varphi$ and new fermion at the electroweak scale after integrating out TeV fermions. The Lagrangian of case (B-1) has $\varphi \varphi \bar{N} N$ interaction, while case (B-2) has $\varphi \bar{N} N$ interaction at the EW scale. So the same high dimensional operator could originate from different UV topology with number of propagators from heavy fermions between cases (B-1) and (B-2).

Temperature dependence. In these cases (B-1) and (B-2), new fermions only contribute to the high dimensional operators with positive size, but cannot give rise to large negative quartic coupling in the potential. Therefore it is difficult to generate a barrier in the potential with this case (B), since the high dimensional operator term is typically much smaller than tree-level ones, and there is no enough negative quartic contribution.

\subsection{One fermion is at $\mathrm{TeV}$ scale and another is at $\mathrm{EW}$ scale}

In this parameter region $\left(m_{L} \gg m_{N} \sim y_{N} v\right), m_{N}$ is at the EW scale and $m_{L}$ is at the TeV scale. The detail of matching and running in the model with this parameter is also in the appendix A. In this case, the effective potential at the EW scale is obtained to be

$$
\begin{aligned}
V_{\mathrm{eff}}^{E W}= & \frac{\mu^{2}}{2} \varphi^{2}+\frac{\lambda}{4} \varphi^{4}+\sum_{i=S M, N_{1}} \frac{n_{i}}{64 \pi^{2}} M_{i}(\varphi)^{4}\left(\log \frac{M_{i}(\varphi)^{2}}{v^{2}}-c_{i}\right) \\
& +\frac{\lambda_{(6)}}{6} \varphi^{6}+\frac{\lambda_{(8)}}{8} \varphi^{8}+\frac{\lambda_{(10)}}{10} \varphi^{10},
\end{aligned}
$$


where the dimensional 6,8 and 10 operators at the EW scale are roughly given by

$$
\begin{aligned}
\lambda_{(6)} & \simeq \frac{m_{L} y_{N}^{6}}{16 \pi^{2}\left(m_{L}-m_{N}\right)^{5}}\left(m_{L}^{2}+7 m_{N} m_{L}-2 m_{N}^{2}\right)-\beta_{6 \varphi} \log \frac{m_{L}}{v} \\
\lambda_{(8)} & \simeq-\frac{y_{N}^{8}}{48 \pi^{2}\left(m_{L}-m_{N}\right)^{7}}\left(7 m_{L}^{3}+27 m_{N} m_{L}^{2}-4 m_{N}^{3}\right)-\beta_{8 \varphi} \log \frac{m_{L}}{v}, \\
\lambda_{(10)} \simeq & \frac{y^{10}}{384 \pi^{2} m_{L}\left(m_{L}-m_{N}\right)^{9}}\left(107 m_{L}^{4}+342 m_{L}^{3} m_{N}+42 m_{L}^{2} m_{N}^{2}-68 m_{L} m_{N}^{3}-3 m_{N}^{4}\right) \\
& -\beta_{10 \varphi} \log \frac{m_{L}}{v},
\end{aligned}
$$

where the first term presents the threshold effect and the second term the running effect. The higher dimensional operators, such as the dimensional 6 operator $\lambda_{(6)}$, in $m_{L} \gg m_{N} \sim$ $y_{N} v$ case are typically larger than other cases in eqs. (3.13), (3.14) due to mass splitting from the mixing effects.

The quartic effective coupling $\lambda_{\text {eff }}^{T=0}$ in this parameter region $(\mathrm{C})$ is roughly given by

$$
\lambda_{\mathrm{eff}}^{T=0} \sim \lambda_{\mathrm{eff}}^{S M}-2 \gamma_{\mathrm{eff}}^{T=0} v^{2}-3 \lambda_{(8)} v^{4}-4 \lambda_{(10)} v^{6}-\frac{y_{N}^{4} m_{N}^{2}}{8 \pi^{2} m_{L}^{2}} \log \frac{m_{N}^{2}}{v^{2}},
$$

where $\gamma_{\text {eff }}^{T=0}$ is $\lambda_{(6)}$ in eq. (3.16) and logarithmic term comes from the loop effect of the new light fermion.

On the other hand, the quadratic effective coupling $\left(\mu_{\mathrm{eff}}^{T=0}\right)^{2}$ in the case $(\mathrm{C})$ is roughly given as

$$
\left(\mu_{\mathrm{eff}}^{T=0}\right)^{2} \sim \lambda_{\mathrm{eff}}^{S M}+\gamma_{\mathrm{eff}}^{T=0} v^{4}+2 \lambda_{(8)} v^{6}+3 \lambda_{(10)} v^{8}+\frac{y_{N}^{2} m_{N}^{3}}{4 \pi^{2} m_{L}} \log \frac{m_{N}^{2}}{v^{2}}
$$

which has the almost same terms as quartic coupling $\lambda_{\text {eff }}^{T=0}$. The quadratic and quartic terms contain not only the light fermion loop effect but also the heavy fermion effect, which in sum have larger positive and negative fermion effects than cases (A) and (B), respectively.

We will focus on this case and perform numerical analyses. Since the terms in the zero temperature serve as the starting point of our analysis, we will first investigate various terms in the zero temperature potential. The numerical results of $\lambda_{\text {eff }}^{T=0}$ and $\gamma_{\text {eff }}^{T=0}$ in $\left(m_{N}, m_{L}\right)$ plane with $y_{N}=2.5,3$ and 4 are shown in figures 2-(1), 2-(2) and 2-(3), respectively. For the RG running analysis, we fixed initial values, for example $y_{N}$ at $Q_{M}$ and $\mu^{2}$ and $\lambda$ at the vacuum expectation value through stationary condition and Higgs boson mass. When there is large loop effect, we cannot realize the exactly initial values by the results of RG running analysis, for example, $\left(\lambda_{(6)}\left(Q_{M}\right)-\gamma_{t h}\right) \neq 0$, where $\lambda(6)\left(Q_{M}\right)$ is the RG running result at $Q_{M}$ and $\gamma_{t h}$ corresponds to the initial value at $Q_{M}$. If there are large differences, we may not explain the Higgs boson mass including the experimental uncertainty: $m_{h}=$ $125.25 \pm 0.17$ [60]. Since this uncertainty for Higgs boson mass appears from $10^{-3}$ orders, we neglect the parameter regions below red line where the difference from the RG running analysis is larger than $10^{-3}$. 

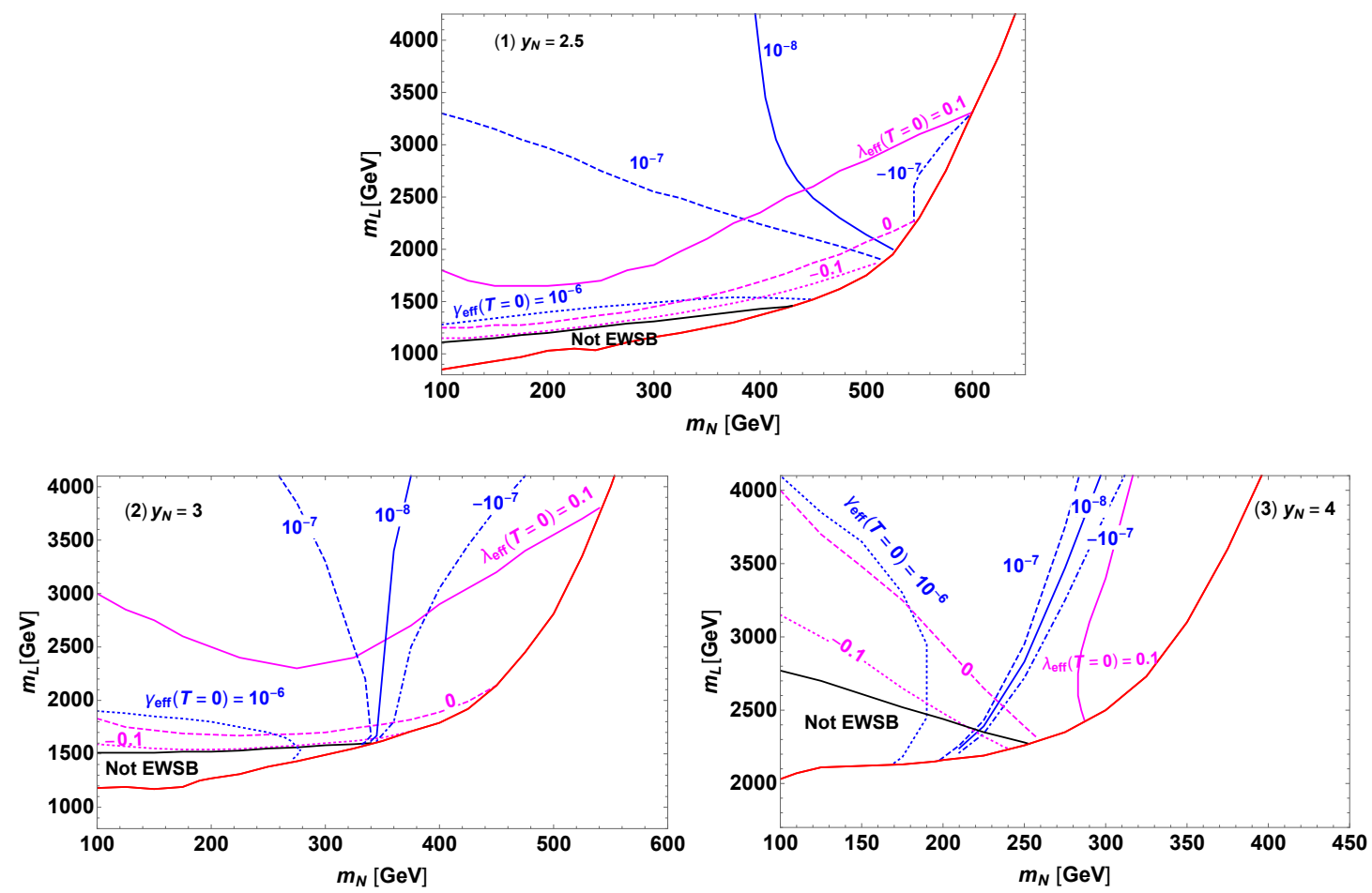

Figure 2. The contours of $\lambda_{\text {eff }}$ and $\gamma_{\text {eff }}$ at zero temperature with $y_{N}=2.5,3$ and 4 in figures 2-(1), 2(2) and 2-(3), respectively. Magenta solid, dashed, dotted lines are $\lambda_{\text {eff }}^{T=0}=0.1,0,-0.1$, respectively. Also, blue dotted, dashed, solid, dashed dotted lines are $\gamma_{\text {eff }}^{(T=0)}=10^{-6}, 10^{-7}, 10^{-8},-10^{-7}$, respectively. The red line represents the criterion that indicates the RG running analysis is appropriate or not, e.g. the condition $\left(\lambda_{(6)}\left(Q_{M}\right)-\gamma_{t h}\right) / \gamma_{t h}<10^{-3}$ (above red line) or $>10^{-3}$ (below red line). Also, symmetry breaking does not occur below the black line.

In the parameter space below the black line, the spontaneous symmetry breaking cannot occur because the minimum of the potential is at origin. Blue lines correspond to contours about the value of $\gamma_{\text {eff }}$ in eq. (3.16). On the other hand, magenta lines represent contour lines of $\lambda_{\text {eff }}$ at zero temperature in eq. (3.17) which have $\gamma_{\text {eff }}$ term.

In order to prove the convergence of the expansion of effective couplings in eq. (3.5), we clarify amounts of normalized effective couplings in figure 3. To define the normalized effective couplings, we used $100 \mathrm{GeV}$ as normalization factor, because a barrier typically shows up in the potential around $\varphi \sim 100 \mathrm{GeV}$. From this figures, the higher normalized effective couplings, the smaller these values become. When $y_{N}$ is large, the figures are in figure 10, the higher coupling, such as $\gamma$ term, becomes zero and is smaller than higher effective couplings, however, the overall values of them decrease. Therefore the expansion of effective couplings can converge within our analysis.

Behaviour of blue contour $\gamma_{\text {eff }}$. According to eq. (3.16), the dimensional 6 operator is roughly given as

$$
\gamma_{\mathrm{eff}}^{T=0} \simeq \frac{y_{N}^{6}}{16 \pi^{2} m_{L}^{2}}\left(1-3\left(\frac{3 y_{t}^{2}}{8 \pi^{2}}+2 \frac{m_{N}}{m_{L}}\right) \log \frac{m_{L}}{v}\right) .
$$



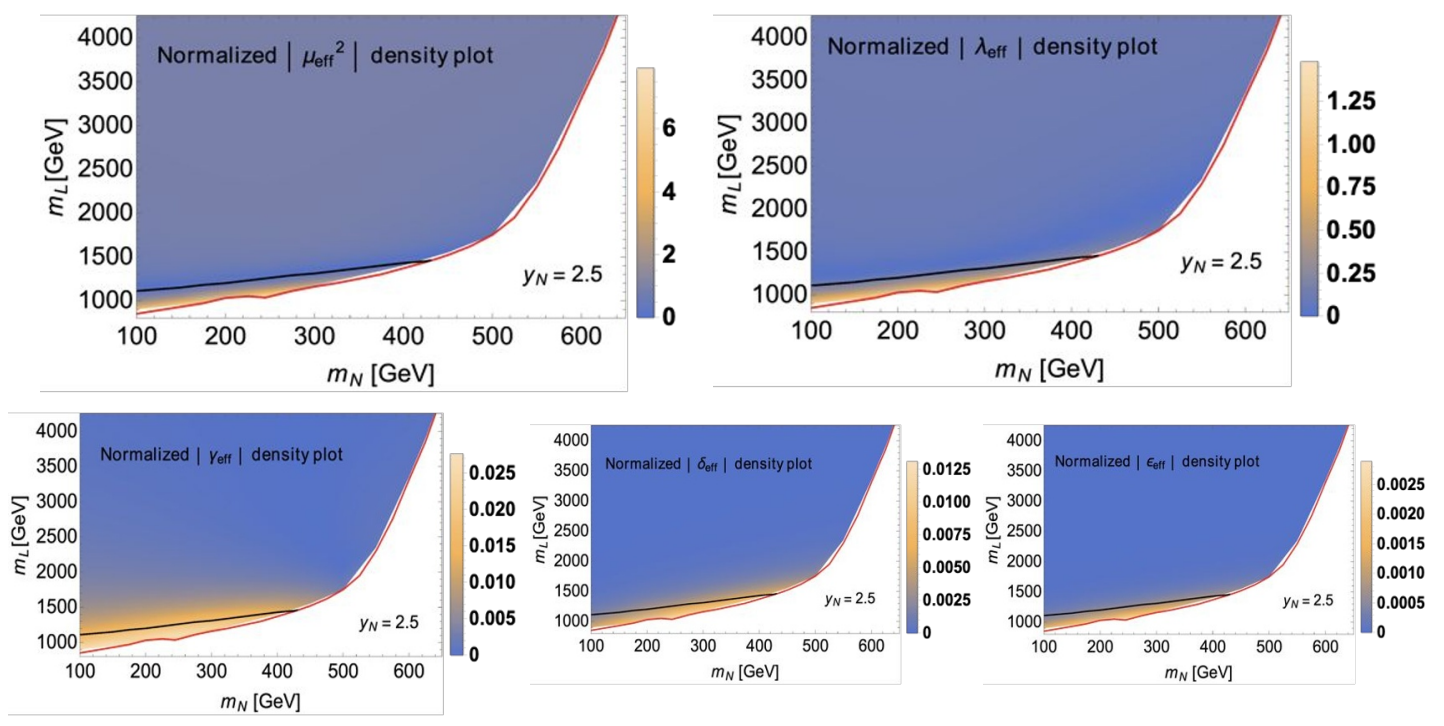

Figure 3. Density plots of absolute values of normalized effective couplings $\mu_{\text {eff }}^{2}, \lambda_{\text {eff }}, \gamma_{\text {eff }}, \delta_{\text {eff }}$ and $\epsilon_{\mathrm{eff}}$ at zero temperature. In this figure, the value of $y_{N}$ is 2.5 and the noramlization factor is $100 \mathrm{GeV}$. Other $y_{N}$ cases are shown in appendix.

From this equation, the $\gamma_{\text {eff }}^{T=0}$ could be negative when $m_{N} / m_{L}$ is large, as shown dashed dotted lines in the figure 2. The negative effect is the source of different behaviours of $\gamma_{\text {eff }}^{T=0}$, especially blue dashed and solid lines, between figure 2-(1) and figures 2-(2), -(3). Since small $m_{L}$ is prohibited by the RG running in figures $2-(2)$, - (3), the ratio of $m_{N} / m_{L}$ can only be increased by large $m_{N}$ value. From that, the slope of blue solid line, for example, change from figure 2-(1) to figures 2-(3).

Behaviour of magenta contour $\boldsymbol{\lambda}_{\text {eff }}$. The quartic term $\lambda_{\text {eff }}^{T=0}$ in eq. (3.17) has not only $\gamma_{\text {eff }}^{T=0}$ but also negative loop effect of light fermion. Since the negative light fermion loop effect in eq. (3.17) is proportional to $\left(m_{N} / m_{L}\right)^{2}$, the $\lambda_{\text {eff }}^{T=0}$ could be small positive or negative value even if $\gamma_{\text {eff }}^{T=0}$ has a negative contribution from RG running term $\left(m_{N} / m_{L}\right) \log m_{L} / v$. Therefore the behaviour of $\lambda_{\text {eff }}^{T=0}$ can have downward convex shapes in figure 2-(1) and 2-(2). In the figure 2-(3), the behaviour of magenta line could be similar to blue line, because the $\gamma_{\text {eff }}^{T=0}$ and the one-loop effect of light fermion are respectively proportional to $y_{N}^{6}$ and $y_{N}^{4}$.

According to the figure 2, the quartic term at the zero temperature potential determines which cases would happen: if the quartic term is positive and comparable to the cubic term, it would be possible to satisfy the scenario (I) discussed in the introduction; if the quartic term is negative, then it is likely to realise the scenario (II). Also, in this parameter region, new fermion effects could cause the potential to be unstable. We discuss the detail of the stability and Landau pole in the appendix B. 
Temperature dependence. From eq. (3.5), new fermion contributions with the $T$ effect in the quadratic and the quartic terms are obtained to be

$$
\begin{aligned}
\left(\mu_{\mathrm{eff}}^{\text {new fermion }}\right)^{2} & \simeq \gamma_{\mathrm{eff}}^{T=0} v^{4}+\frac{y_{N}^{2} X}{2(1-X)}\left(-\frac{T^{2}}{3}+\frac{X^{2} m_{L}^{2}}{2 \pi^{2}}\left(\ln \frac{\alpha_{F} T^{2}}{v^{2}}-\frac{3}{2}\right)\right), \\
\lambda_{\text {eff }}^{\text {new fermion }} & \simeq-2 \gamma_{\text {eff }}^{T=0} v^{2}+\frac{4 y_{N}^{4}(1+X)}{16 m_{L}^{2}(1-X)^{3}}\left(\frac{T^{2}}{3}-\frac{X^{2} m_{L}^{2}(3-X)}{2 \pi^{2}(1+X)}\left(\ln \frac{\alpha_{F} T^{2}}{v^{2}}-\frac{3}{2}\right)\right),
\end{aligned}
$$

where $X \equiv m_{N} / m_{L}$. From the above equation, we note that the larger the mixing effect, which is proportional to $y_{N} X$, the more negative quartic coupling in the Higgs potential. At the same time, in the quadratic term, if the mixing effect becomes larger, the positive logarithmic term dominates over the negative $T^{2}$ term, and verse vice. The finite temperature effect on the dimensional 6 term is

$$
\gamma_{\text {eff }}^{\text {T,fermion }} \simeq \frac{y_{N}^{6}(1+X)}{16 m_{L}^{4}(1-X)^{5}}\left(-2 T^{2}+\frac{3 m_{L}^{2} X}{\pi^{2}}\left(\ln \frac{\alpha_{F} T^{2}}{v^{2}}-\frac{3}{2}\right)\right) .
$$

Similarly the larger the mixing effects, the larger the higher dimensional term contribution.

So beside the size and sign of the quartic term at the zero temperature, the mixing effects, parametrized by the $y_{N} X$ also control whether the sizable barrier could be realized.

- In the scenario (II), it needs negative quartic term and large mixing angle. In this scenario, at low temperature comparable to the EW scale, these logarithmic terms dominate the effective couplings in the quadratic and quartic terms: the quadratic term is enhanced by the new fermion positive logarithmic term besides the SM quadratic $T^{2}$ contribution, while the quartic coupling is further decreased by the new fermion negative logarithmic term. A large barrier could be generated through the positive quadratic and sextic effective couplings $\left(\mu_{\text {eff }}^{2}, \gamma_{\text {eff }}\right)$ and the negative quartic one $\left(\lambda_{\text {eff }}\right)$.

- On the other hand, if the mixing effects are not so large, and the quartic coupling at zero temperature is small but still positive, the scenario (I) could be realized. In this scenario, since the mixing is not so large the negative $T^{2}$ term dominates over the logarithmic term in the quadratic term. Thus there is cancellation between the negative $T^{2}$ term from the fermions and the SM thermal contribution, and thus the quadratic term is decreased to smaller size which is comparable to the cubic term in the SM. In total, the effective potential with comparable positive quadratic, negative cubic and positive quartic terms could realize first-order EWPT.

In summary, the extended fermion model with this mass region could generate a barrier through the (I) positive $\varphi^{2}$ term, negative $\varphi^{3}$ term and positive $\varphi^{4}$ term and the (II) positive $\varphi^{2}$ term, negative $\varphi^{4}$ term and positive higher dimensional term scenarios.

\section{Numerical results on phase transition pattern}

As discussed above, in cases (A) and (B), it is difficult to generate a sizable barrier and thus cannot realize first-order phase transition. In the following, we will focus on the case (C) 


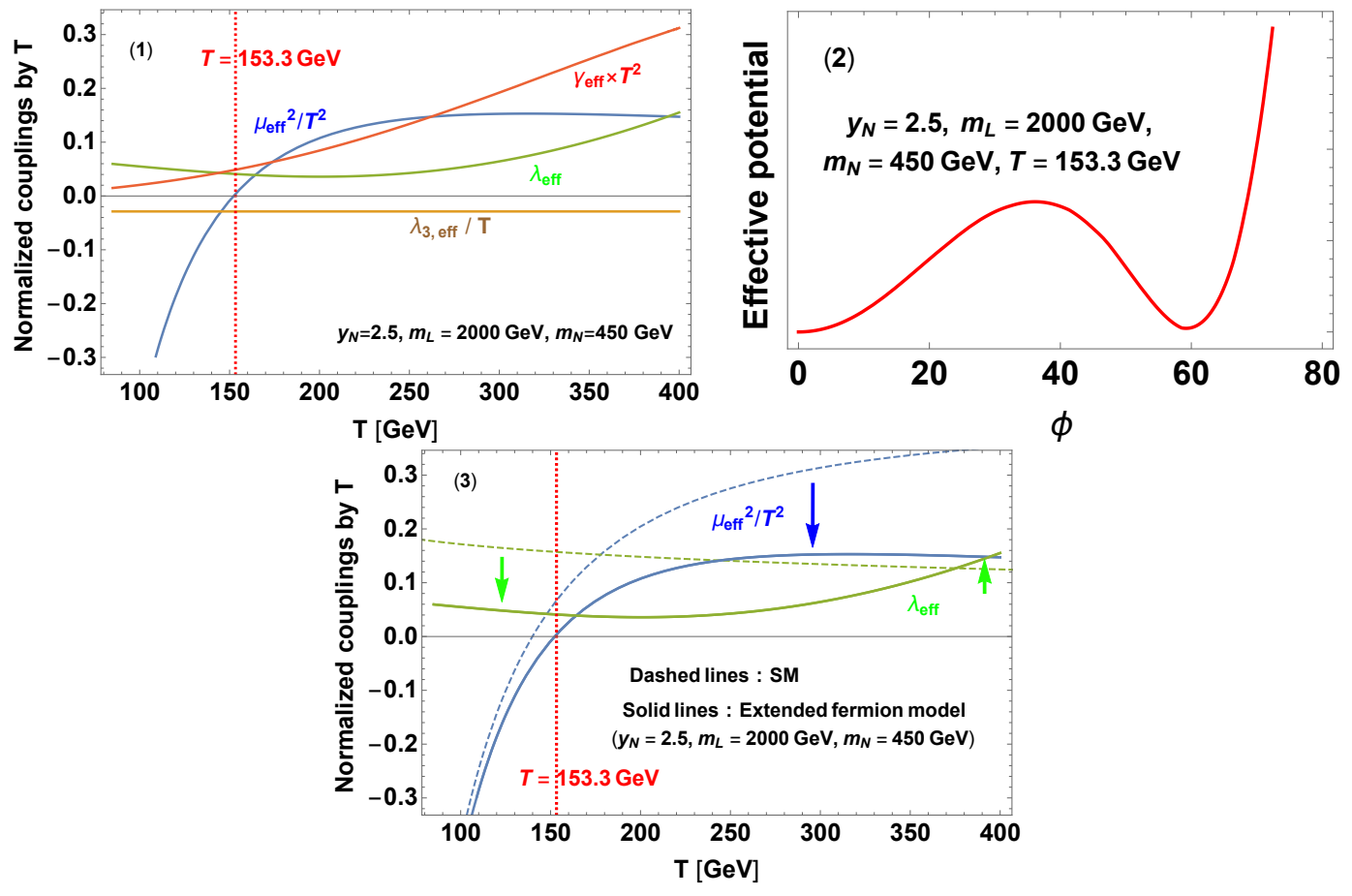

Figure 4. Upper left figure 4-(1) represents the $T$ dependence of normalized effective couplings of the model with $y_{N}=2.5, m_{L}=2000 \mathrm{GeV}$ and $m_{N}=450 \mathrm{GeV}$ : blue $\mu_{\text {eff }}^{2} / T^{2}$, brawn $\lambda_{3, e f f} / T$, green $\lambda_{\text {eff }}$ and red $\gamma_{\mathrm{eff}} T^{2}$. The red dotted lines in figure 4-(1) and lower one 4-(3) correspond to the critical temperature of the model with this benchmark point: $T=153.3 \mathrm{GeV}$. Upper right figure 4-(2) describe the shape of potential at the critical temperature. Lower figure 4-(3) represents differences of quadratic (blue) and quartic (green) couplings between in the extended fermion model with this benchmark point (solid lines) and in the SM (dashed line).

to explore how to generate a barrier under the scenarios (I) and (II). We will numerically analyze the phase transition pattern by using both the high temperature approximation and exact thermal effective potential. There are three new parameters $\left(y_{N}, m_{L}, m_{N}\right)$ in the model. Before we perform a parameter scan over all the parameters, we first analyze the phase transition scenarios with chosen benchmark points.

First, let us discuss the scenario (I) with the benchmark point: $y_{N}=2.5, m_{L}=$ $2000 \mathrm{GeV}$ and $m_{N}=450 \mathrm{GeV}$. The $T$ dependence of normalized effective couplings in eq. (3.5) with this benchmark point are shown in figure 4-(1). The blue, brawn, green and red lines in the figure correspond to the $T$ dependences of $\mu_{\text {eff }}^{2} / T^{2}, \lambda_{3, \text { eff }} / T, \lambda_{\text {eff }}$ and $\gamma_{\mathrm{eff}} T^{2}$, respectively. In figure 4-(2), the shape of the effective potential is described at critical temperature, at which there is degenerate vacuum in the potential. The red dotted lines in upper right figure 4-(1) and in lower figure 4-(3) represents the critical temperature of the model with this benchmark point, $T=153.3 \mathrm{GeV}$. From figure $4-(1)$, we note that the normalized effective couplings are almost the same size around the critical temperature (red dotted line) and then a sizable barrier is developed. Lower figure 4-(3) shows the difference in $T$ dependence of normalized effective couplings $\mu_{\text {eff }}^{2} / T^{2}$ and $\lambda_{\text {eff }}$ between the extended fermion model with the benchmark point (solid lines) and the SM (dashed lines). 

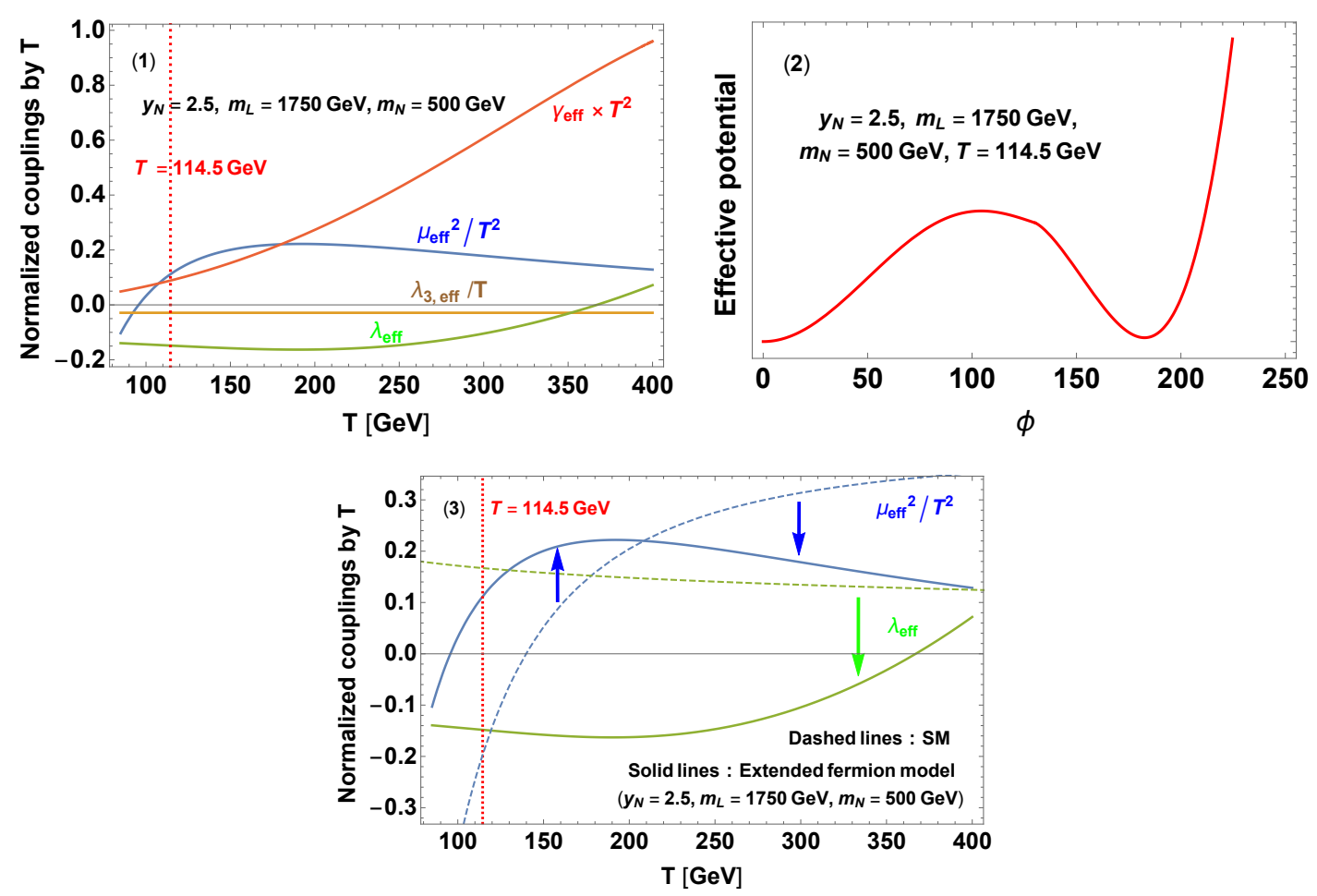

Figure 5. The case of extended fermion model with $y_{N}=2.5, m_{L}=1750 \mathrm{GeV}$ and $m_{N}=$ $500 \mathrm{GeV}$. The model with this benchmark point could generate a barrier by scenario (II). The critical temperature about this benchmark point is $T=114.5 \mathrm{GeV}$. Otherwise the same as figure 4 .

Compared to the SM, the normalized effective couplings are quite different: $\mu_{\text {eff }}^{2} / T^{2}$ and $\lambda_{\text {eff }}$ are decreased compared to the SM values. Arrows in figure 4-(3) represent the amount of change from the SM to the extended fermion model with this benchmark point. The directions of arrows change at low temperature, because then the logarithmic term of $T$ becomes dominant contribution.

Next, we discuss the scenario (II) with another benchmark point $y_{N}=2.5, m_{L}=$ $1750 \mathrm{GeV}$ and $m_{N}=500 \mathrm{GeV}$. Figure 5 represents results for this benchmark point by the same analyses as figure 4 . This benchmark point has larger a value of $m_{N} / m_{L}$ than one for the scenario (I) in figure 4, and then the fermionic reduction contributions of last terms in eqs. (3.20)-(3.22) become large. Therefore the behaviours of normalized effective couplings except for $\lambda_{e f f, 3} / T$ in figure 5 are different from ones in figure 4 . Since the normalized quartic coupling $\lambda_{\text {eff }}$ becomes negative around a critical temperature $T=114.5 \mathrm{GeV}$ for the model with this benchmark point, a barrier could be developed through the scenario (II).

Now we start to scan over possible parameter regions and perform numerical calculation on the exact thermal Higgs potential. In practice, we adopt the following strategies: (1) in the scenario I, the quadratic term is nearly zero, while the quartic term is still positive; (2) in the scenario II, the only requirement is the negative quartic coupling near the critical temperature. In order to check the parameter region where a barrier is generated through 

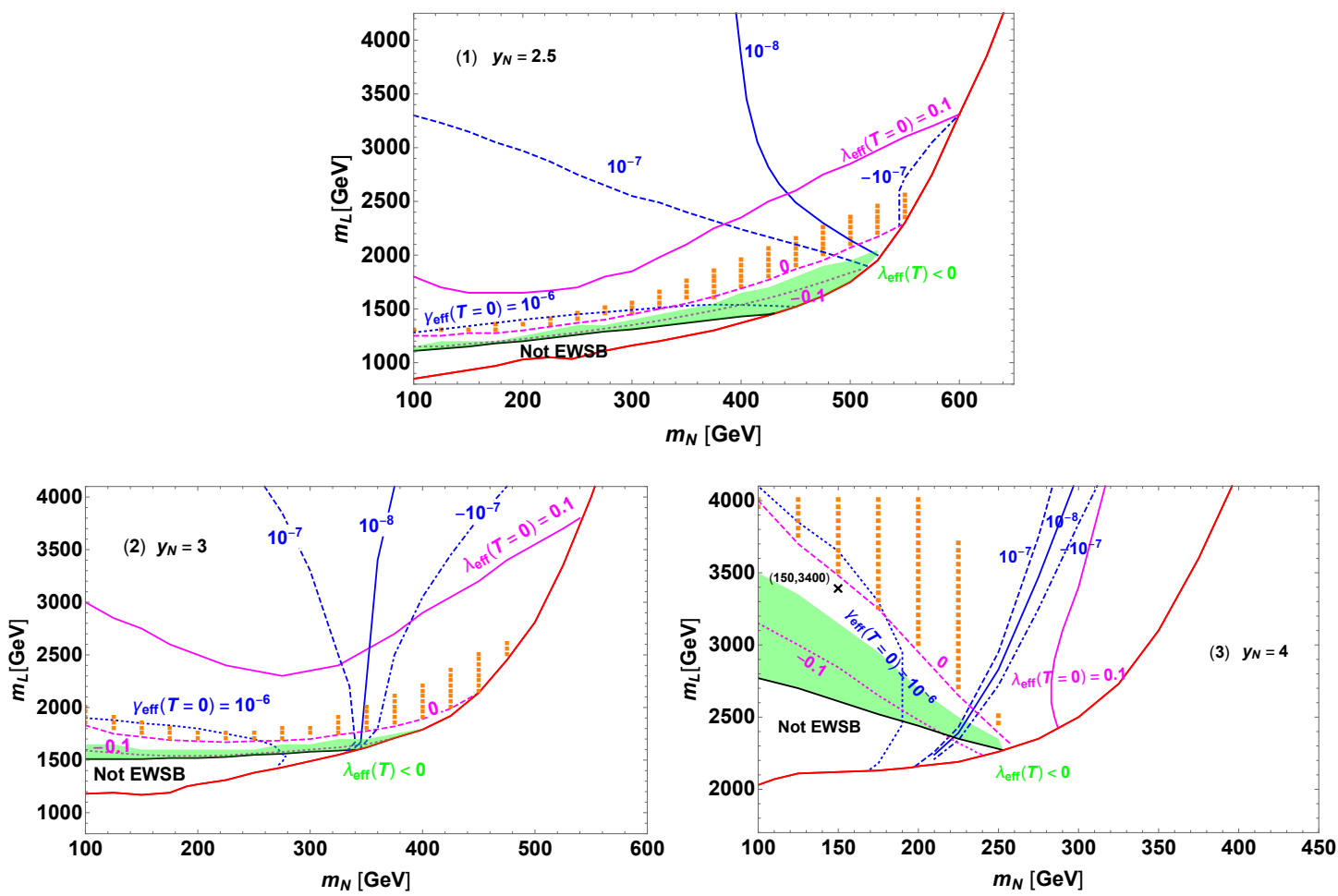

Figure 6. The region represents the parameters which could generate a barrier through the scenarios (I) and (II). The contours are the same as figure 2. Orange square mark region can satisfy the conditions in eq. (4.1) [scenario (I)]. In the green region, negative $\lambda_{\text {eff }}(T)$ can be achieved [scenario (II)]. Black cross mark is one of example in the vacant region between the orange mark region and the green region. At this black cross mark, the first-order EWPT can be realized in the scenario (I) (figure 7).
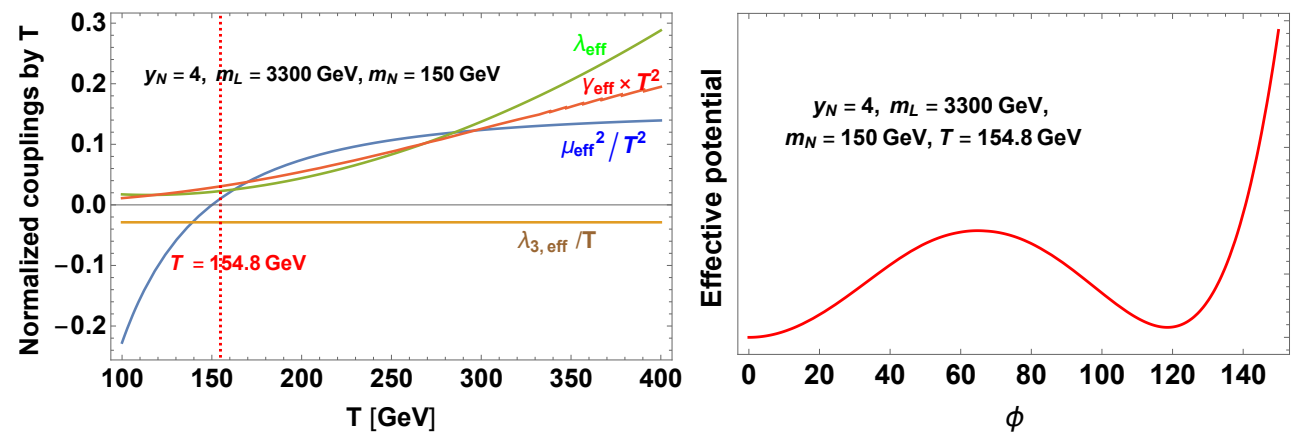

Figure 7. Left: the $T$ dependence of normalized effective couplings in the extended fermion model at the black cross mark in figure $6\left(y_{N}=4, m_{L}=3300 \mathrm{GeV}\right.$ and $\left.m_{N}=150 \mathrm{GeV}\right)$. Right: the shape of the effective potential in the model with this benchmark point at $T=154.8 \mathrm{GeV}$, which is a red dotted line in left figure. 
the scenario (I) more precisely, we explore a temperature $T$ by the following strategy:

$$
\frac{\mu_{\text {eff }}^{2}}{T^{2}}=0, \quad 0.7<\frac{\lambda_{\text {eff }}}{\frac{\lambda_{3, e f f}}{T}}<3, \quad \lambda_{\text {eff }}^{T=0}>0 .
$$

From figure 4-(1), the scenario (I) requires $\mu_{\text {eff }}^{2} / T^{2} \sim 0$, almost the same sizes of $\lambda_{\text {eff }}$ and $\lambda_{3, e f f} / T$ and positive quartic coupling $\lambda_{\text {eff }}$ to generate a sizable barrier. Therefore we use the conditions in eq. (4.1) to represent the parameter region where a sizable barrier is developed through the scenario (I). Also, to find the model parameters $\left(y_{N}, m_{N}, m_{L}\right)$ for generating a barrier via the scenario (II), we numerically analyze the parameter space where negative $\lambda_{\text {eff }}(T)$ appears in $T=[10,246] \mathrm{GeV}$ per $10 \mathrm{GeV}$. For the parameter scan, we use $m_{L}=[1000,4000] \mathrm{GeV}$ per $50 \mathrm{GeV}$ and $m_{N}=[100,700] \mathrm{GeV}$ per $25 \mathrm{GeV}$. The results of numerical analysis for the scenarios (I) and (II) are shown in figure 6 . The orange square marks in this figure are parameters satisfying the condition about the scenario (I) in eq. (4.1). The green regions represent $\lambda_{\text {eff }}(T)<0$. Otherwise the same as figure 2. There are the vacant regions between the green region and the orange mark in figure 6. A sizable barrier could be developed in these vacant regions, for example, figure 7 shows the $T$ dependence of the normalized couplings at a black cross mark in figure 6-(3). According to the figure 7, the black cross mark could have a barrier through the scenario (I). The different point between vacant region and orange square marks is the sign of quartic coupling at zero temperature $\lambda_{\text {eff }}^{T=0}$, however, in the vacant region, this coupling is positive tiny amount around the critical temperature like figure 7. Although the quartic coupling becomes negative at zero temperature, the Higgs potential is always stable. The detail is discussed in appendix B.

From figure 6, a barrier could be generated in the case that $\lambda_{\text {eff }}^{T=0}$ is small positive value or negative value, especially around magenta dashed line. Such a value of $\lambda_{\text {eff }}^{T=0}$ can be achieved by the large $\gamma_{\text {eff }}^{T=0}$ (second term in eq. (3.17)) or the large one-loop effect of the new light fermion (last term in eq. (3.17)). The scenario (II) (green region) typically requires larger $m_{N}$ or smaller $m_{L}$ than the scenario (I) (orange squared marks) when $y_{N} \leq 3$. In the case that $y_{N}=4$, because the behaviour of magenta line $\lambda_{\text {eff }}^{T=0}$ could be similar to blue line $\gamma_{\mathrm{eff}}^{T=0}$, the behaviours of orange marks and green region in figure $6-1,-2$ are different from ones in figure 6-3.

According to figure 6 , let us discuss the value of $\varphi_{C} / T_{C}$ for each parameter point, where $T_{C}$ is critical temperature and $\varphi_{C}$ is the minimum of the potential at the $T_{C}$. The $\varphi_{C} / T_{C}$ provides one key criterions whether the electroweak baryogenesis scenario could be successfully realizing the baryon asymmetry of the universe. Roughly, the criterion is estimated as $\varphi_{C} / T_{C}>1$. We show the density plots in figure 8 which is related to this condition. This condition can be satisfied in scenario (II), because the EWPT in the scenario is related to the tree-level effects and a barrier could be generated at low temperature.

Finally, we discuss experimental constraints and testability in the parameter regions in figure 6. Because there is one light stable neutral fermion $N_{1}$ at the EW scale, there are some experimental constraints, such as the invisible $Z$ decay, the invisible $h$ decay and direct search of dark matter [58]. For large values of $y_{N}$, a sizable barrier could be easily 

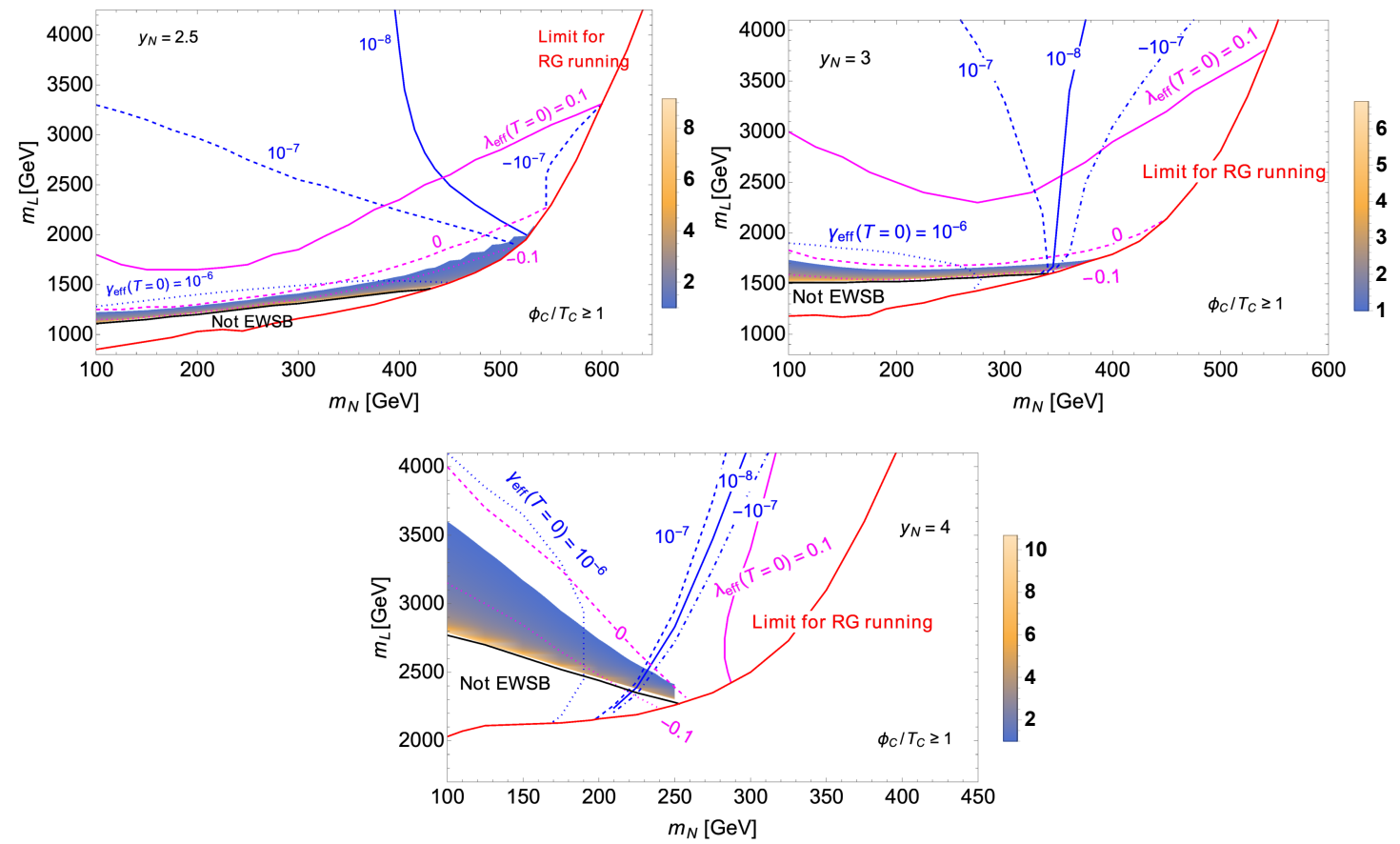

Figure 8. Density plots which satisfy the condition $\varphi_{C} / T_{C}>1$ for each parameter point, in the parameter space with $y_{N}=2.5,3$ and 4 . Otherwise the same as figure 2 .

developed in the potential, however, on the other hand, the constraint from the direct search for dark matter becomes strong. Therefore, we consider the mixing term between the SM particle and the new fermion: $\epsilon_{N^{\prime}} \bar{L}_{\tau} H N^{\prime}$ and $\epsilon_{L} \bar{L} H \tau$ where $L_{\tau}^{T}=\left(\tau, \nu_{\tau}\right)$ and $\tau$ are related to the third generation of the SM lepton. The light neutral fermion $N_{1}$ can decay to $W^{*} \tau$ by this mixing effect. Then a doublet fermion in the mass range of $120-790 \mathrm{GeV}$, which couples to the third generation leptons, is excluded at $95 \%$ confidence level [59]. In our analyses in figure 6, we set the doublet fermion mass to $O(1 \mathrm{TeV})$ to take these experimental data into account, and we also take the mixing effects $\epsilon_{N^{\prime}}$ and $\epsilon_{L}$ to be tiny amounts in order to make sure the shape of effective potential does not change much and the $N_{1}$ particle is unstable.

The parameter regions where a barrier could be developed in figure 6 could be tested by the precision measurements of the triple Higgs boson coupling $\lambda_{h h h}$. The ratio of $\lambda_{h h h}$ between the extended fermion model and the SM prediction is defined as

$$
\kappa_{\lambda}=\frac{\lambda_{h h h}}{\lambda_{h h h}^{S M}}
$$

where $\lambda_{h h h}$ and $\lambda_{h h h}^{S M}$ are the triple Higgs boson coupling in the extended fermion model and in the SM, which is obtained as

$$
\left.\lambda_{h h h} \equiv \frac{\partial^{3} V_{\mathrm{eff}}}{\partial \varphi^{3}}\right|_{\varphi=v} .
$$



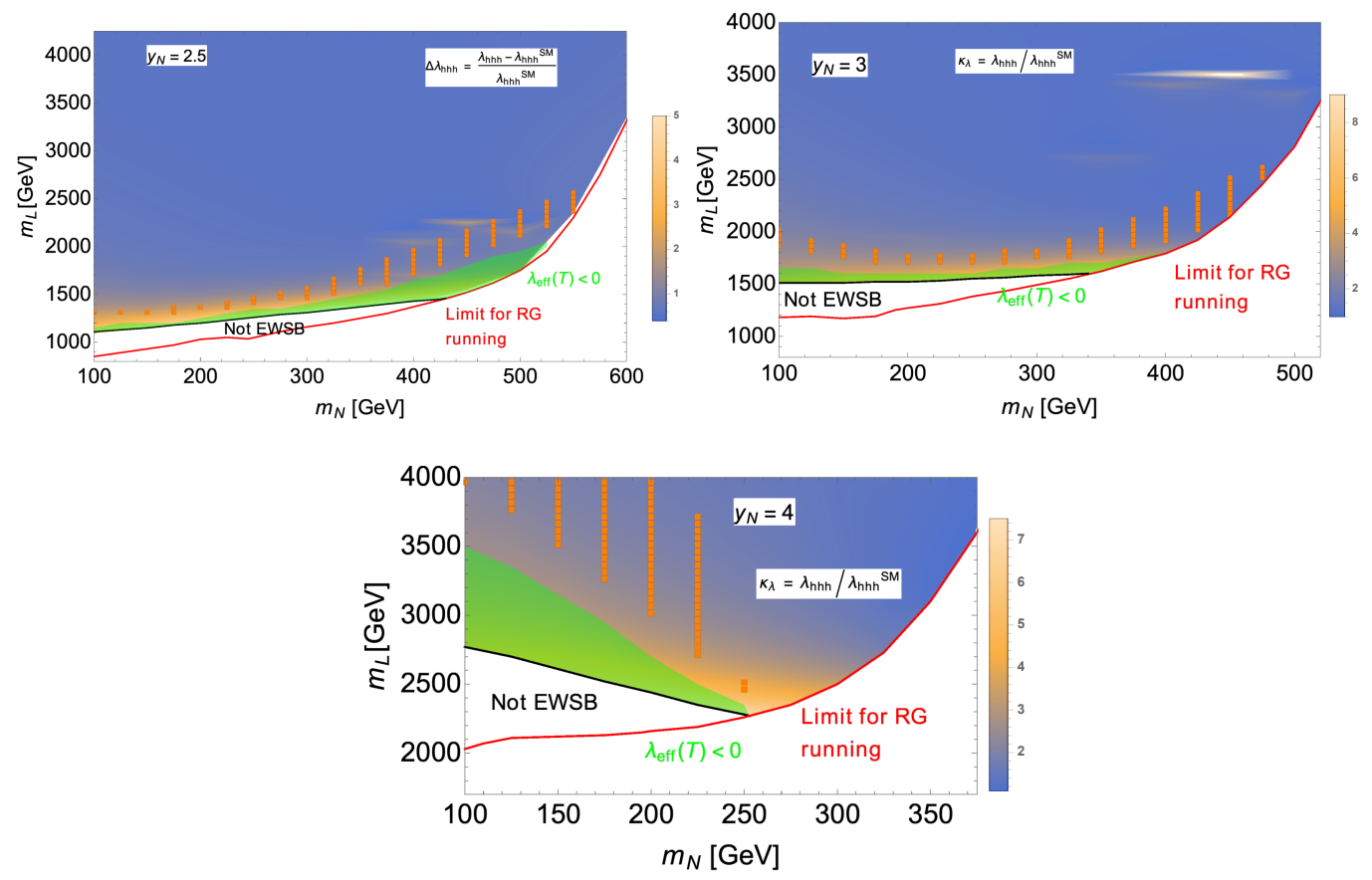

Figure 9. Density plots of the ratio of triple Higgs boson coupling between the extended fermion model and the SM prediction $\kappa_{\lambda}=\lambda_{h h h} / \lambda_{h h h}^{S M}$. The Orange square mark region and the green region are the same as figure 6 and the black and the red lines are the same as figure 2 .

Figure 9 represents the value of $\kappa_{\lambda}$ for the parameter region in figure 6 . To understanding the behaviours of density plots in the figures, we show the analytical results for new fermion contributions from eq. (3.15) to the hhh coupling. Based on eqs. (3.15) and (4.3), the new fermion effects in the $h h h$ coupling is roughly

$$
\lambda_{h h h}^{\text {new fermion }} \sim 8 \gamma v^{3}+\frac{y_{N}^{6} v^{3} X}{\pi^{2} m_{L}^{2}(1-X)^{2}\left(1-X-\frac{y_{N}^{2} v^{2}}{X m_{L}^{2}}\right)},
$$

and thus the value of $h h h$ coupling could be enhanced by large mass ratio $X=m_{N} / m_{L}$. In the case of large $y_{N}$, the $h h h$ coupling in eq. (4.4) could be further reduced to

$$
\lambda_{h h h}^{\text {new fermion }} \sim 8 \gamma v^{3}-\frac{y_{N}^{4} v X^{2}}{\pi^{2}(1-X)^{2}} \quad\left(\text { large } y_{N}\right) .
$$

The $y_{N}$ dependence of second term in the $h h h$ coupling with large $y_{N}$ becomes $y_{N}^{4}$, which is smaller than $\gamma \sim y_{N}^{6}$ coming from eq. (3.19). In such a case, the behaviour of $h h h$ is similar to one of $\gamma_{\mathrm{eff}}(T=0)$ in eq. (3.19), which is enhanced by large $m_{L}$ or small $m_{N}$. These analytical results of the $h h h$ coupling can match density plots of $\kappa_{\lambda}$ in figure 9 .

The color region of the ratio of $h h h$ coupling in figure 9 represents the allowed regio by the current experimental data: $\kappa_{\lambda}>-2.3$ and $10.3<\kappa_{\lambda}$ are excluded at $95 \%$ confidence level [61]. Especially, the $h h h$ coupling in the orange square marks and green region in figure 6 could be as large as 10\% more than the SM prediction value. Such large deviation in the $h h h$ coupling can be measured at future collider experiments [62-66], and thus we could check whether a first-order EWPT can be realized or not by measurements of the hhh coupling. 


\section{Summary}

First-order electroweak phase transition is one of the crucial ingredients to realize the baryon asymmetry of the universe in electroweak baryogenesis scenario. Usually it could be triggered by enhanced contribution to the cubic term in the Higgs potential from the bosonic degree of freedom. Although fermionic degree of freedom does not contribute to cubic term in the potential, it is still possible to develop a sizable barrier in the effective potential via either decreasing quadratic/quartic terms or adding higher dimensional operators. In this work, we investigated the phase transition patterns in various fermionic extensions of the standard model, and realized it is necessary to introduce multi-fermions. For simplicity, we take the simplest model with more than one fermion, one doublet fermion and singlet neutral fermion.

In this model we classified the mass pattern of the fermions into three regions: (A) both fermions are at the EW scale, (B) both fermions are at the TeV scale and (C) one is at the EW scale and another is at the $\mathrm{TeV}$ scale. We found that both cases (A) and (B) could not generate a sizable barrier, while case $(\mathrm{C})$ could generate one. Also, in case $(\mathrm{C})$ we found that such a barrier can be developed if the Higgs potential satisfies two scenarios: (I) positive quadratic term $\mu_{\text {eff }}^{2}$, negative cubic term $\lambda_{3, \text { eff }}$ and positive quartic term $\lambda_{\text {eff }}$ or (II) positive quadratic term $\mu_{\text {eff }}^{2}$, negative quartic term $\lambda_{\text {eff }}$ and positive high dimensional operator, such as dimensional 6 operator $\gamma_{\text {eff. }}$. In these scenarios, comparable size of new fermions could contribute to these effective couplings through high dimensional operators of heavy fermion and loop effect of light fermion. Depending on the mixing angles between new fermions and the size and sign of the quartic coupling at the zero temperature, some parameter regions in case (C) receive positive and negative contributions from additional fermions to $\mu_{\mathrm{eff}}^{2}$ and $\lambda_{\text {eff }}$ terms, respectively. These effects are the source of a barrier regarding scenarios (I) or (II) and increase by large value of $m_{N} / m_{L}$.

In this work, we utilize the effective field theory approach to treat the multi-scale effective potential of the two fermions model via the matching and running procedure. This treatment is quite general and can be extended to other new physics models with heavy fields and light fields, not limited to the fermion degree of freedom. Thus this general effective potential with matching and running is easy to apply to any other models with multi-scales involved in.

\section{Acknowledgments}

K.H. is grateful to Prof. Shinya Kanemura for helpful discussions. J.H.Y. is supported in part by National Key Research and Development Program of China Grant No. 2020YFC2201501, and the National Science Foundation of China (NSFC) under Grants No. 12022514, No. 11875003 and No. 12047503, and CAS Project for Young Scientists in Basic Research YSBR-006, and the Key Research Program of the CAS Grant No. XDPB15. Q.H.C. is supported in part by the National Science Foundation of China under Grant Nos. 11725520, 11675002, 11635001. 


\section{A Derivation of the effective potential in case B and C and normalized effective couplings}

In this appendix, we discuss the detail of the treatment of the effective potential with multi-scales in section III.

\section{A.1 Case B-1: $m_{L} \gg m_{N} \gg y_{N} v$}

In this parameter, we consider two-step matchings: (i) very HE scale $m_{L}$ and HE scale $m_{N}$ and (ii) the HE scale and LE scale. The Lagrangian at the very HE scale is eq. (3.1). The field dependent masses are the same as eq. (3.3). The very HE and HE effective potentials at high matching scale $Q_{M, \text { heavy }}$ are

$$
\begin{aligned}
V_{\mathrm{eff}}^{\text {very } H E}= & \frac{\mu^{2}}{2} \varphi^{2}+\frac{\lambda}{4} \varphi^{4}+\sum_{i=N_{1}, N_{2}, S M} \frac{n_{i}}{64 \pi^{2}} M_{i}(\varphi)^{4}\left(\log \frac{M_{i}(\varphi)^{2}}{Q_{M, \text { heavy }}^{2}}-c_{i}\right) \\
V_{\mathrm{eff}}^{H E}= & \frac{\left(\mu^{2}\right)^{H E}}{2} \varphi^{2}+\frac{\lambda^{H E}}{4} \varphi^{4}+\sum_{i=N_{1}, S M} \frac{n_{i}}{64 \pi^{2}} M_{i}(\varphi)^{4}\left(\log \frac{M_{i}(\varphi)^{2}}{Q_{M, \text { heavy }}^{2}}-c_{i}\right) \\
& +\frac{\gamma^{H E}}{6} \varphi^{6}+\frac{\delta^{H E}}{8} \varphi^{8}+\frac{\epsilon^{H E}}{10} \varphi^{10} .
\end{aligned}
$$

By using the matching and running procedure in section 2, the parameters in the HE scale potential at $Q_{M, h e a v y}$ are given as

$$
\begin{aligned}
\left(\mu^{2}\right)^{H E} & =\mu^{2}+\frac{y_{N}^{2} m_{L}^{3}}{4 \pi^{2}\left(m_{L}-m_{N}\right)}, \quad \lambda^{H E}=\lambda-\frac{m_{L}^{2}\left(m_{L}+m_{N}\right) y_{N}^{4}}{8 \pi^{2}\left(m_{L}-m_{N}\right)^{3}}, \\
\gamma^{H E} & =\frac{m_{L} y_{N}^{6}\left(m_{L}^{2}+7 m_{L} m_{N}-2 m_{N}^{2}\right)}{16 \pi^{2}\left(m_{L}-m_{N}\right)^{5}}, \quad \delta^{H E}=-\frac{y_{N}^{8}\left(7 m_{L}^{3}+27 m_{L}^{2} m_{N}-4 m_{N}^{3}\right)}{48 \pi^{2}\left(m_{L}-m_{N}\right)^{7}}, \\
\epsilon^{H E} & =-\frac{y_{N}^{10}\left(107 m_{L}^{4}+342 m_{L}^{3} m_{N}+42 m_{L}^{2} m_{N}^{2}-68 m_{L} m_{N}^{3}-3 m_{N}^{4}\right)}{384 \pi^{2} m_{L}\left(m_{L}-m_{N}\right)^{9}}
\end{aligned}
$$

where we set $Q_{M \text {, heavy }}$ as $m_{L}$. The RG running effect from the HE scale $Q_{H E}$ to the LE scale $Q_{L E}$ roughly is

$$
\beta_{n \varphi}=\frac{d \lambda_{(n)}}{d \log \mu} \quad \rightarrow \quad \lambda_{(n)}^{R G} \simeq \int_{Q_{L E}}^{Q_{H E}} \beta_{n \varphi} d \log \mu=-\beta_{n \varphi} \log \frac{Q_{H E}}{Q_{L E}},
$$

In order to obtain the beta function, we treat the flavor state as mass eigenstate, and then the Lagrangian of new fermion at the HE scale is given by

$$
-\mathcal{L}_{V L L}^{H E} \supset \bar{N}_{1}\left(-i \not \partial+m_{N}\right) N_{1}-M_{2 \varphi}^{N} \bar{N}_{1} N_{1}\left(\varphi^{2}+\varphi_{z}^{2}+2 \varphi^{+} \varphi^{-}\right)
$$

where $N_{1}$ is mass eigenstate of light fermion and

$$
M_{2 \varphi}^{N}=\frac{y_{N}^{2}}{2\left(m_{L}-m_{N}\right)} .
$$


By using the Lagrangian of eq. (A.4), the beta functions of dimensional 6, 8 and 10 are

$$
\begin{aligned}
& \beta_{6 \varphi}^{H E}=\frac{3 m_{N}\left(M_{2 \varphi}^{N}\right)^{3}}{\pi^{2}}-6 \frac{\gamma^{H E}}{16 \pi^{2}}\left(-3 y_{t}^{2}+\frac{3}{4} g_{1}^{2}+\frac{9}{4} g_{2}^{2}\right), \\
& \beta_{8 \varphi}^{H E}=-\frac{\left(M_{2 \varphi}^{N}\right)^{4}}{\pi^{2}}-8 \frac{\delta^{H E}}{16 \pi^{2}}\left(-3 y_{t}^{2}+\frac{3}{4} g_{1}^{2}+\frac{9}{4} g_{2}^{2}\right), \\
& \beta_{10 \varphi}^{H E}=-10 \frac{\epsilon^{H E}}{16 \pi^{2}}\left(-3 y_{t}^{2}+\frac{3}{4} g_{1}^{2}+\frac{9}{4} g_{2}^{2}\right) .
\end{aligned}
$$

The second terms in the dimensional 6 and 8 operators and the right-hand side of dimensional 10 operator come from $\gamma_{\varphi}$ function in eq. (2.18). Furthermore, the first terms in the dimensional 6 and 8 operators can be obtained by the first term in eq. (2.18). The SM fields, such as the gauge boson and the top quark, do not contribute to these operators through the first term in eq. (2.18). The dimensional 6,8 and 10 operators at low matching scale $Q_{M, L E}$, which is related to second step matching (ii) the HE scale and the LE scale, are roughly

$$
\begin{gathered}
\gamma^{H E, M}=\gamma^{H E}-\beta_{6 \varphi}^{H E} \log \frac{m_{L}}{Q_{M, L E}}, \quad \delta^{H E, M}=\delta^{H E}-\beta_{8 \varphi}^{H E} \log \frac{m_{L}}{Q_{M, L E}} \\
\epsilon^{H E, M}=\epsilon^{H E}-\beta_{10 \varphi}^{H E} \log \frac{m_{L}}{Q_{M, L E}}
\end{gathered}
$$

The HE and LE effective potentials at $Q_{M, L E}$ are

$$
\begin{aligned}
V_{\mathrm{eff}}^{H E}= & \frac{\left(\mu^{2}\right)^{H E, M}}{2} \varphi^{2}+\frac{\lambda^{H E, M}}{4} \varphi^{4}+\sum_{i=N_{1}, S M} \frac{n_{i}}{64 \pi^{2}} M_{i}(\varphi)^{4}\left(\log \frac{M_{i}(\varphi)^{2}}{Q_{M, L E}^{2}}-c_{i}\right) \\
& +\frac{\gamma^{H E, M}}{6} \varphi^{6}+\frac{\delta^{H E, M}}{8} \varphi^{8}+\frac{\epsilon^{H E, M}}{10} \varphi^{10} . \\
V_{\mathrm{eff}}^{L E}= & \frac{\left(\mu^{2}\right)^{L E}}{2} \varphi^{2}+\frac{\lambda^{L E}}{4} \varphi^{4}+\sum_{i=S M} \frac{n_{i}}{64 \pi^{2}} M_{i}(\varphi)^{4}\left(\log \frac{M_{i}(\varphi)^{2}}{Q_{M, L E}^{2}}-c_{i}\right) \\
& +\frac{\gamma^{L E}}{6} \varphi^{6}+\frac{\delta^{L E}}{8} \varphi^{8}+\frac{\epsilon^{L E}}{10} \varphi^{10} .
\end{aligned}
$$

In the following, we set $Q_{M, L E}$ as $m_{N}$. The LE parameters at this scale are obtained by the matching conditions:

$$
\begin{aligned}
\left(\mu^{2}\right)^{L E} & =\left(\mu^{2}\right)^{H E, M}-\frac{y_{N}^{2} m_{N}^{3}}{4 \pi^{2}\left(m_{L}-m_{N}\right)}, \quad \lambda^{L E}=\lambda^{H E, M}+\frac{m_{N}^{2}\left(m_{L}+m_{N}\right) y_{N}^{4}}{8 \pi^{2}\left(m_{L}-m_{N}\right)^{3}}, \\
\gamma^{L E} & =\gamma^{H E, M}+\frac{m_{N} y_{N}^{6}\left(2 m_{L}^{2}-7 m_{L} m_{N}-m_{N}^{2}\right)}{16 \pi^{2}\left(m_{L}-m_{N}\right)^{5}} \\
\delta^{H E} & =\delta^{H E, M}-\frac{y_{N}^{8}\left(4 m_{L}^{3}-27 m_{L} m_{N}^{2}-7 m_{N}^{3}\right)}{48 \pi^{2}\left(m_{L}-m_{N}\right)^{7}} \\
\epsilon^{L E} & =\epsilon^{H E, M}+\frac{y_{N}^{10}\left(3 m_{L}^{4}+68 m_{L}^{3} m_{N}-42 m_{L}^{2} m_{N}^{2}-342 m_{L} m_{N}^{3}-107 m_{N}^{4}\right)}{384 \pi^{2} m_{N}\left(m_{L}-m_{N}\right)^{9}} .
\end{aligned}
$$


The Lagrangian at the LE scale is the same as the SM, so the beta functions of the high dimensional operators are

$$
\begin{aligned}
& \beta_{6 \varphi}^{L E}=-6 \frac{\gamma^{L E}}{16 \pi^{2}}\left(-3 y_{t}^{2}+\frac{3}{4} g_{1}^{2}+\frac{9}{4} g_{2}^{2}\right), \quad \beta_{8 \varphi}^{L E}=-8 \frac{\delta^{L E}}{16 \pi^{2}}\left(-3 y_{t}^{2}+\frac{3}{4} g_{1}^{2}+\frac{9}{4} g_{2}^{2}\right) \\
& \beta_{10 \varphi}^{L E}=-10 \frac{\epsilon^{H E}}{16 \pi^{2}}\left(-3 y_{t}^{2}+\frac{3}{4} g_{1}^{2}+\frac{9}{4} g_{2}^{2}\right) .
\end{aligned}
$$

By using them, we can obtain the effective potential at EW scale in eq. (2.19).

\section{A.2 Case B-2: $m_{L} \sim m_{N} \gg y_{N} v$}

In this parameter case, we need one-step matching-running between HE and LE scale to integrate out additional doublet and singlet fermions. The HE and LE effective potentials at the matching scale $Q_{M}$ are

$$
\begin{aligned}
V_{\mathrm{eff}}^{H E}= & \frac{\mu^{2}}{2} \varphi^{2}+\frac{\lambda}{4} \varphi^{4}+\sum_{i=N_{1}, N_{2}, S M} \frac{n_{i}}{64 \pi^{2}} M_{i}(\varphi)^{4}\left(\log \frac{M_{i}(\varphi)^{2}}{Q^{2}}-c_{i}\right) \\
V_{\mathrm{eff}}^{L E}= & \frac{\left(\mu^{2}\right)^{L E}}{2} \varphi^{2}+\frac{\lambda^{L E}}{4} \varphi^{4}+\sum_{i=S M} \frac{n_{i}}{64 \pi^{2}} M_{i}(\varphi)^{4}\left(\log \frac{M_{i}(\varphi)^{2}}{Q^{2}}-c_{i}\right) \\
& +\frac{\gamma^{L E}}{6} \varphi^{6}+\frac{\delta^{L E}}{8} \varphi^{8}+\frac{\epsilon^{L E}}{10} \varphi^{10} .
\end{aligned}
$$

The parameters of the potential at the LE scale can be obtained by the matching conditions:

$$
\begin{aligned}
& \left(\mu^{2}\right)^{L E}=\mu^{2}+\frac{y_{N}^{2} m_{L}^{2}}{4 \pi^{2}}, \quad \lambda^{L E}=\lambda-\frac{y_{N}^{4}}{3 \pi^{2}}, \quad \gamma^{L E}=\frac{y_{N}^{6}}{160 \pi^{2} m_{L}^{2}}, \\
& \delta^{L E}=\frac{y_{N}^{8}}{2240 \pi^{2} m_{L}^{4}}, \quad \epsilon^{L E}=\frac{y_{N}^{10}}{16128 \pi^{2} m_{L}^{6}} .
\end{aligned}
$$

where we set the matching scale $Q_{M}$ as $m_{L}$. At the LE scale, the Lagrangian only has the SM fields. Therefore the beta function of high dimensional operators are the same as eq. (A.10):

$$
\begin{aligned}
& \beta_{6 \varphi}=-6 \frac{\gamma^{L E}}{16 \pi^{2}}\left(-3 y_{t}^{2}+\frac{3}{4} g_{1}^{2}+\frac{9}{4} g_{2}^{2}\right), \quad \beta_{8 \varphi}=-8 \frac{\delta^{L E}}{16 \pi^{2}}\left(-3 y_{t}^{2}+\frac{3}{4} g_{1}^{2}+\frac{9}{4} g_{2}^{2}\right) \\
& \beta_{10 \varphi}=-10 \frac{\epsilon^{L E}}{16 \pi^{2}}\left(-3 y_{t}^{2}+\frac{3}{4} g_{1}^{2}+\frac{9}{4} g_{2}^{2}\right) .
\end{aligned}
$$

By using them, we can obtain the effective potential at EW scale in eq. (2.19). 


\section{A.3 Case C: $m_{L} \gg m_{N} \sim y_{N} v$}

In this case, $m_{L}$ is at the TeV scale and $m_{N}$ is at the EW scale. The effective potentials at the HE and the LE scale are

$$
\begin{aligned}
V_{\mathrm{eff}}^{H E}= & \frac{\mu^{2}}{2} \varphi^{2}+\frac{\lambda}{4} \varphi^{4}+\sum_{i=N_{1}, N_{2}, S M} \frac{n_{i}}{64 \pi^{2}} M_{i}(\varphi)^{4}\left(\log \frac{M_{i}(\varphi)^{2}}{Q^{2}}-c_{i}\right) \\
V_{\mathrm{eff}}^{L E}= & \frac{\left(\mu^{2}\right)^{L E}}{2} \varphi^{2}+\frac{\lambda^{L E}}{4} \varphi^{4}+\sum_{i=N_{1}, S M} \frac{n_{i}}{64 \pi^{2}} M_{i}(\varphi)^{4}\left(\log \frac{M_{i}(\varphi)^{2}}{Q^{2}}-c_{i}\right) \\
& +\frac{\gamma^{L E}}{6} \varphi^{6}+\frac{\delta^{L E}}{8} \varphi^{8}+\frac{\epsilon^{L E}}{10} \varphi^{10}
\end{aligned}
$$

and the field dependent masses are the same as eq. (3.3). At the matching scale $Q_{M}$, the parameters $\varphi^{n}$ terms at the HE scale match ones at the LE scale by the matching conditions:

$$
\begin{aligned}
\left(\mu^{2}\right)^{L E} & =\mu^{2}+\frac{y_{N}^{2} m_{L}^{3}}{4 \pi^{2}\left(m_{L}-m_{N}\right)}, \quad \lambda^{L E}=\lambda-\frac{y_{N}^{4} m_{L}^{2}\left(m_{L}+m_{N}\right)}{8 \pi^{2}\left(m_{L}-m_{N}\right)^{3}}, \\
\gamma^{L E} & =\gamma^{t h}, \quad \delta^{L E}=\delta^{t h}, \quad \epsilon^{L E}=\epsilon^{t h},
\end{aligned}
$$

where we set $Q_{M}$ as $m_{L}$. The threshold effects of high dimensional operators $\gamma^{\text {th }}, \delta^{\text {th }}$ and $\epsilon^{\text {th }}$ are given by

$$
\begin{aligned}
\gamma^{\text {th }} & =\frac{m_{L} y_{N}^{6}}{16 \pi^{2}\left(m_{L}-m_{N}\right)^{5}}\left(m_{L}^{2}+7 m_{N} m_{L}-2 m_{N}^{2}\right), \\
\delta^{t h} & =-\frac{y_{N}^{8}}{48 \pi^{2}\left(m_{L}-m_{N}\right)^{7}}\left(7 m_{L}^{3}+27 m_{N} m_{L}^{2}-4 m_{N}^{3}\right), \\
\epsilon^{t h} & =\frac{y_{N}^{10}}{384 \pi^{2} m_{L}\left(m_{L}-m_{N}\right)^{9}}\left(107 m_{L}^{4}+342 m_{L}^{3} m_{N}+42 m_{L}^{2} m_{N}^{2}-68 m_{L} m_{N}^{3}-3 m_{N}^{4}\right),
\end{aligned}
$$

The threshold effects of $\gamma^{t h}$ and $\delta^{t h}$ are the same ones in ref. [21]. In order to obtain the beta functions, we use the Lagrangian in eq. (A.4). The beta functions of dimensional 6, 8 and 10 operators are the same as eq. (A.6):

$$
\begin{aligned}
\beta_{6 \varphi} & =\frac{3 m_{N}\left(M_{2 \varphi}^{N}\right)^{3}}{\pi^{2}}-6 \frac{\gamma^{t h}}{16 \pi^{2}}\left(-3 y_{t}^{2}+\frac{3}{4} g_{1}^{2}+\frac{9}{4} g_{2}^{2}\right), \\
\beta_{8 \varphi} & =-\frac{\left(M_{2 \varphi}^{N}\right)^{4}}{\pi^{2}}-8 \frac{\delta^{t h}}{16 \pi^{2}}\left(-3 y_{t}^{2}+\frac{3}{4} g_{1}^{2}+\frac{9}{4} g_{2}^{2}\right), \\
\beta_{10 \varphi} & =-10 \frac{\epsilon^{t h}}{16 \pi^{2}}\left(-3 y_{t}^{2}+\frac{3}{4} g_{1}^{2}+\frac{9}{4} g_{2}^{2}\right) .
\end{aligned}
$$

By using them, we can obtain the effective potential at EW scale in eq. (2.19). 

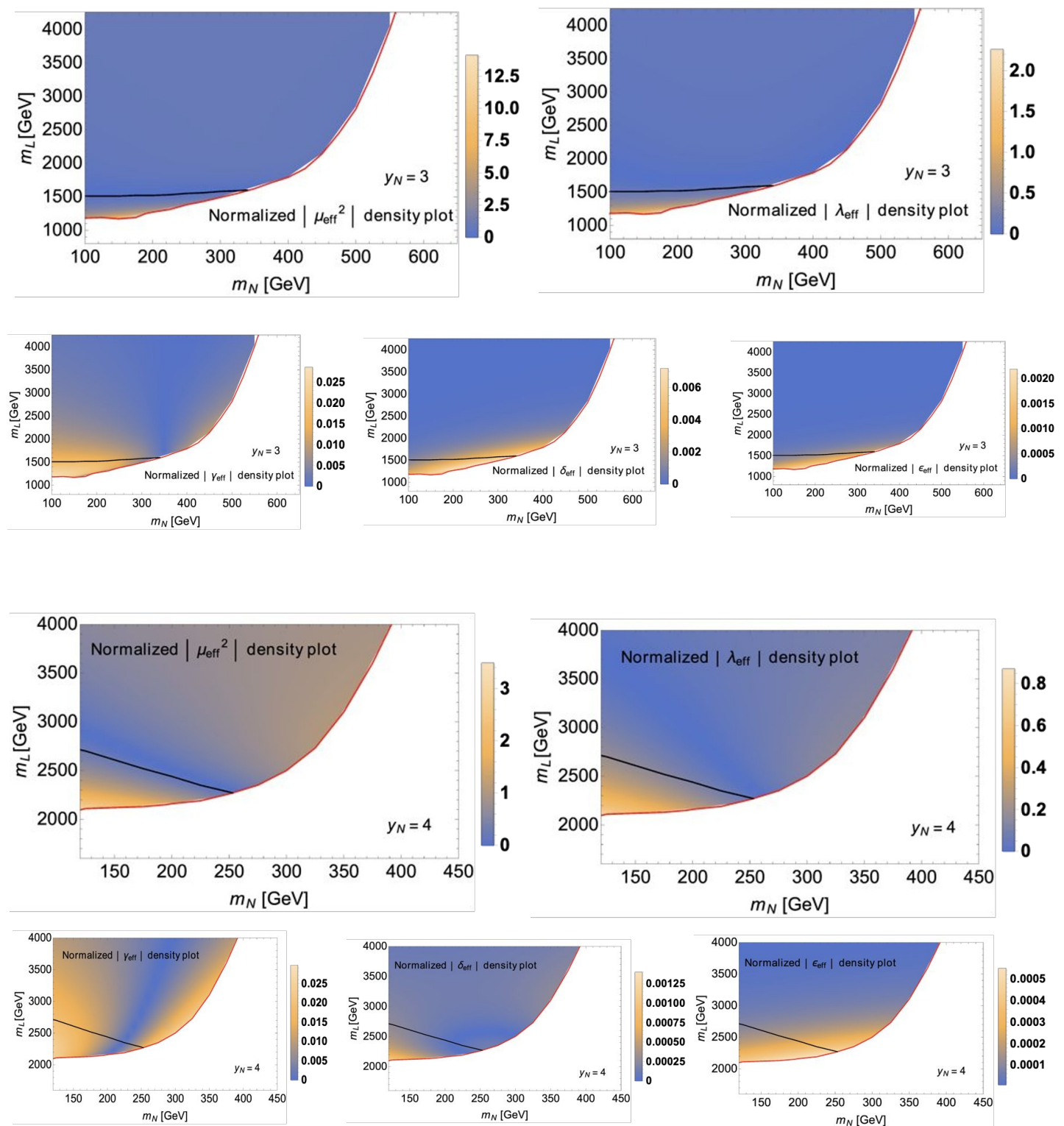

Figure 10. Density plots of absolute values of normalized effective couplings in the model with $y_{N}=3$ and 4 . Otherwise the same as figure 3 .

\section{A.4 Normalized effective couplings in the model with $y_{N}=3$ and 4 in case C}

In figure 3, we show the amounts of normalized effective couplings in the case $\mathrm{C}$ model with $y_{N}=2.5$. When $y_{N}$ value is large, the normalized effective couplings are given as figure 10. In these cases, the higher coupling, such as $\gamma$ term, becomes negative, and then it is smaller than higher effective couplings. However, the overall values of the normalized effective couplings decrease. Therefore the expansion of effective couplings can converge within our analysis. 

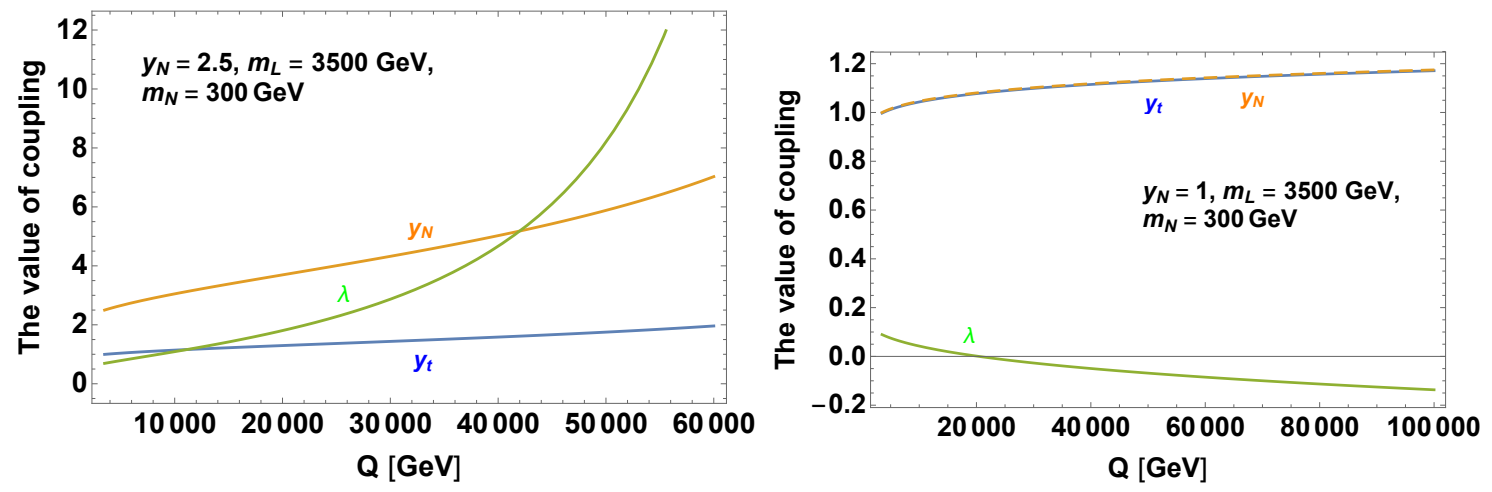

Figure 11. The results of RG running flows of the $y_{t}, y_{N}$ and $\lambda$ couplings in the extended fermion model with $m_{N}=300 \mathrm{GeV}, m_{L}=3500 \mathrm{GeV}$ and $y_{N}=2.5$ (left figure) and $y_{N}=1$ (right figure) from matching scale $Q_{M}=m_{L}$ to $60 \mathrm{TeV}$ (left figure) and to $100 \mathrm{TeV}$ (right figure). Green line: $\lambda$ coupling, yellow line: $y_{N}$ coupling, blue line: $y_{t}$ coupling. In right figure, the yellow and blue lines are the almost same and $\lambda$ coupling becomes negative.

\section{B Vacuum stability and Landau pole}

In this section, we discuss the vacuum stability and the Landau pole $\Lambda_{L}$ in the parameter $(\mathrm{C}): m_{L} \gg m_{N} \sim y_{N} v$. Although $\lambda_{\text {eff }}^{T=0}$ becomes negative in the parameter space in figure 6 , the effective potential at the EW scale is stable by positive high dimensional operators. But, at the HE scale, the potential could become unstable, so we discuss the vacuum stability at the HE scale. The condition of unstable potential at the HE scale is

$$
\lambda^{H E}(Q)<0,
$$

where $\lambda^{H E}(Q)$ is a coefficient of $\varphi^{4}$ in the Higgs potential at HE scale. We focus on the dominant contributions coming from $y_{t}, y_{N}$ and $\lambda$ in order to get the energy scale $\Lambda_{\text {stable }}$ : $\lambda\left(\Lambda_{\text {stable }}\right)=0$. The beta functions of these couplings at the HE scale are

$$
\begin{aligned}
& \beta_{y_{t}}^{H E}=\frac{y_{t}}{16 \pi^{2}}\left(\frac{9 y_{t}^{2}}{2}+2 y_{N}^{2}\right), \quad \beta_{y_{N}}^{H E}=\frac{y_{N}}{16 \pi^{2}}\left(\frac{7 y_{N}^{2}}{2}+3 y_{t}^{2}\right) \\
& \beta_{\lambda}^{H E}=\frac{1}{16 \pi^{2}}\left(-6 y_{t}^{4}-2 y_{N}^{4}+24\left(\lambda^{H E}\right)^{2}-4\left(-3 y_{t}^{2}-2 y_{N}^{2}\right) \lambda^{H E}\right) .
\end{aligned}
$$

We note that the quartic coupling in the HE scale $\lambda^{H E}$ at the matching scale is given by

$$
\lambda^{H E}=\lambda^{L E}+\frac{y_{N}^{4} m_{L}^{2}\left(m_{L}+m_{N}\right)}{8 \pi^{2}\left(m_{L}-m_{N}\right)^{3}}
$$

from eq. (A.15). The second term in the right-hand side of this equation represents the threshold effect in eq. (A.15) which is positive. If the quartic coupling at the matching scale is small positive value, such as the value of the SM case $\lambda \sim 0.1$, the quartic coupling $\lambda$ becomes negative above $\Lambda_{\text {stable }}$, in other words, the shape of the potential becomes unstable. Figure 11 shows an example of the RG running flow of the $y_{t}, y_{N}$ and $\lambda$ couplings. Left panel represents the flows in the extended fermion model with $m_{N}=300 \mathrm{GeV}, m_{L}=3500 \mathrm{GeV}$ 

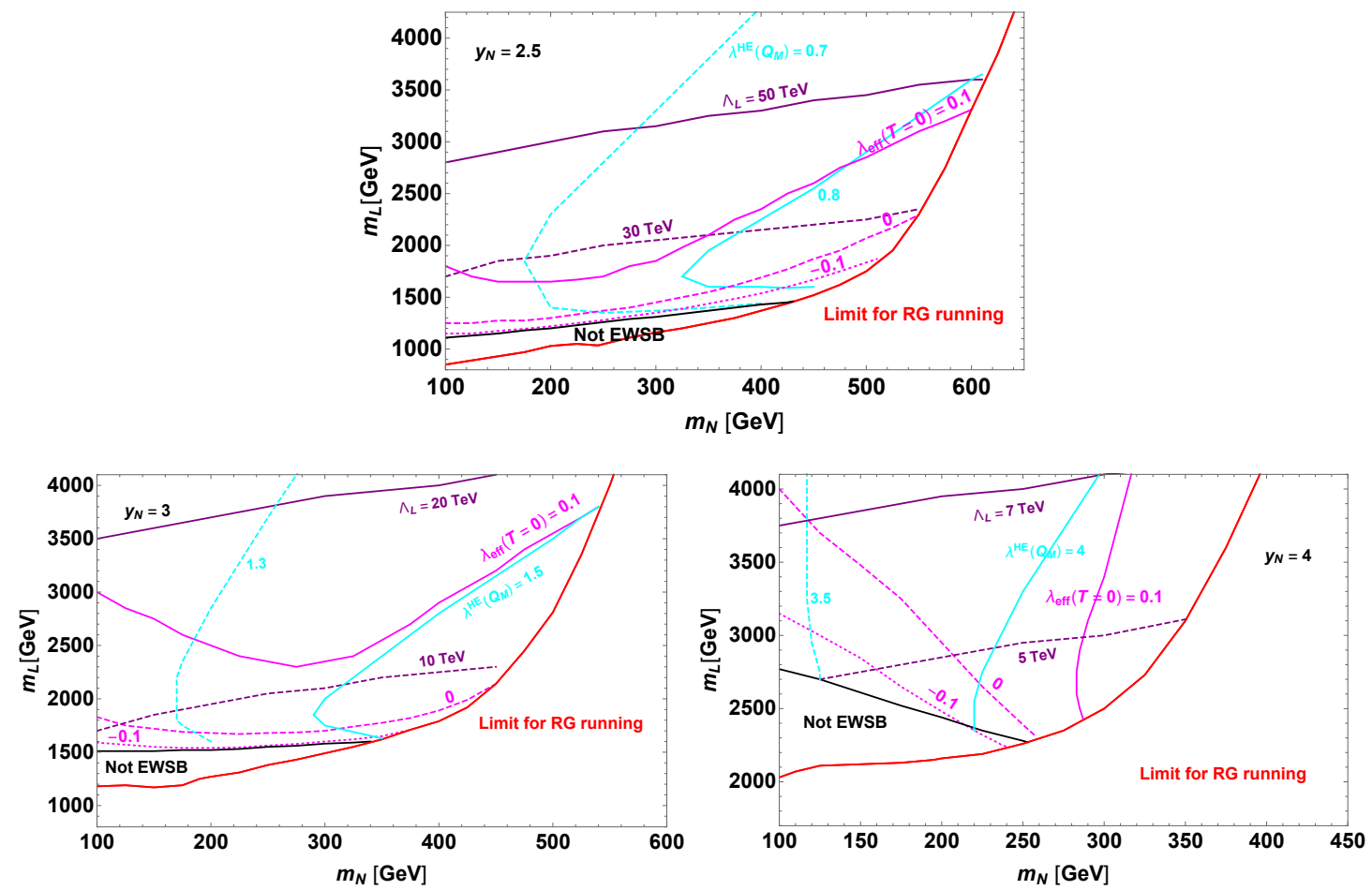

Figure 12. Cyan contours represent the value of $\lambda^{H E}$ in eq. (B.3) at matching scale $Q_{M}=m_{L}$ and purple contours represent value of landau pole $\Lambda_{L}$, where one of coupling is $4 \pi$ at this energy scale, in the extended fermion model with $y_{N}=2.5$ (upper), 3 (lower left) and 4 (lower right). Magenta contours, black and red lines are the same as figure 2.

and $y_{N}=2.5$, while right panel has different $y_{N}$ value from left panel: $m_{N}=300 \mathrm{GeV}$, $m_{L}=3500 \mathrm{GeV}$ and $y_{N}=1$. The right panel has $\Lambda_{\text {stable }}$, because the value of $\lambda^{H E}$ in the panel at the matching scale is similar to one of the SM case: $\lambda \sim 0.1$. In this case, the negative fermion effects in the RG running are dominant and $\lambda^{H E}$ becomes negative

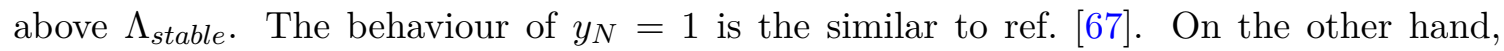
the quartic coupling in the left panel at the matching scale is larger than the value of SM case by additional positive contribution coming from second term of eq. (B.3), and then the potential is stable not only at LE scale but also at HE scale. The value of $\lambda^{H E}$ at the matching scale $Q_{M}$ represents the cyan lines in figure 12. From this figure, the $\lambda^{H E}\left(Q_{M}\right)$ coupling in the parameter region of our analysis is larger than the SM value and the potential is always stable.

Furthermore, we analyze the Landau pole $\Lambda_{L}$ at which a coupling is $4 \pi$. The landau pole represents purple lines in figure 12 . When the value of $m_{L}$ is small, the Landau pole $\Lambda_{L}$ becomes small. The value of $\Lambda_{L}$ is always larger than the matching scale $Q_{M}=m_{L}$.

Open Access. This article is distributed under the terms of the Creative Commons Attribution License (CC-BY 4.0), which permits any use, distribution and reproduction in any medium, provided the original author(s) and source are credited. 


\section{References}

[1] ATLAS collaboration, Observation of a new particle in the search for the Standard Model Higgs boson with the ATLAS detector at the LHC, Phys. Lett. B 716 (2012) 1 [arXiv:1207.7214] [INSPIRE].

[2] CMS collaboration, Observation of a New Boson at a Mass of $125 \mathrm{GeV}$ with the CMS Experiment at the LHC, Phys. Lett. B 716 (2012) 30 [arXiv:1207.7235] [InSPIRE].

[3] P. Agrawal, D. Saha, L.-X. Xu, J.-H. Yu and C.P. Yuan, Determining the shape of the Higgs potential at future colliders, Phys. Rev. D 101 (2020) 075023 [arXiv:1907.02078] [INSPIRE].

[4] V.A. Kuzmin, V.A. Rubakov and M.E. Shaposhnikov, On the Anomalous Electroweak Baryon Number Nonconservation in the Early Universe, Phys. Lett. B 155 (1985) 36 [InSPIRE].

[5] A. Kosowsky, M.S. Turner and R. Watkins, Gravitational radiation from colliding vacuum bubbles, Phys. Rev. D 45 (1992) 4514 [InSPIRE].

[6] A. Kosowsky, M.S. Turner and R. Watkins, Gravitational waves from first order cosmological phase transitions, Phys. Rev. Lett. 69 (1992) 2026 [InSPIRE].

[7] C. Grojean and G. Servant, Gravitational Waves from Phase Transitions at the Electroweak Scale and Beyond, Phys. Rev. D 75 (2007) 043507 [hep-ph/0607107] [InSPIRE].

[8] S.J. Huber and T. Konstandin, Gravitational Wave Production by Collisions: More Bubbles, JCAP 09 (2008) 022 [arXiv:0806.1828] [INSPIRE].

[9] C. Caprini, R. Durrer and G. Servant, The stochastic gravitational wave background from turbulence and magnetic fields generated by a first-order phase transition, JCAP 12 (2009) 024 [arXiv:0909.0622] [INSPIRE].

[10] P. Binetruy, A. Bohe, C. Caprini and J.-F. Dufaux, Cosmological Backgrounds of Gravitational Waves and eLISA/NGO: Phase Transitions, Cosmic Strings and Other Sources, JCAP 06 (2012) 027 [arXiv: 1201.0983] [INSPIRE].

[11] C. Caprini et al., Science with the space-based interferometer eLISA. II: Gravitational waves from cosmological phase transitions, JCAP 04 (2016) 001 [arXiv:1512.06239] [INSPIRE].

[12] M. Dine, R.G. Leigh, P. Huet, A.D. Linde and D.A. Linde, Comments on the electroweak phase transition, Phys. Lett. B 283 (1992) 319 [hep-ph/9203201] [InSPIRE].

[13] K. Kajantie, M. Laine, K. Rummukainen and M.E. Shaposhnikov, The Electroweak phase transition: A Nonperturbative analysis, Nucl. Phys. B 466 (1996) 189 [hep-lat/9510020] [INSPIRE].

[14] L. Dolan and R. Jackiw, Symmetry Behavior at Finite Temperature, Phys. Rev. D 9 (1974) 3320 [INSPIRE].

[15] M. Pietroni, The Electroweak phase transition in a nonminimal supersymmetric model, Nucl. Phys. B 402 (1993) 27 [hep-ph/9207227] [INSPIRE].

[16] M. Carena, A. Megevand, M. Quirós and C.E.M. Wagner, Electroweak baryogenesis and new TeV fermions, Nucl. Phys. B 716 (2005) 319 [hep-ph/0410352] [INSPIRE].

[17] M. Fairbairn and P. Grothaus, Baryogenesis and Dark Matter with Vector-like Fermions, JHEP 10 (2013) 176 [arXiv:1307.8011] [INSPIRE].

[18] A. Aranda, E. Jiménez and C.A. Vaquera-Araujo, Electroweak phase transition in a model with gauged lepton number, JHEP 01 (2015) 070 [arXiv: 1410.7508] [INSPIRE]. 
[19] D. Egana-Ugrinovic, The minimal fermionic model of electroweak baryogenesis, JHEP 12 (2017) 064 [arXiv:1707.02306] [INSPIRE].

[20] A. Angelescu and P. Huang, Multistep Strongly First Order Phase Transitions from New Fermions at the TeV Scale, Phys. Rev. D 99 (2019) 055023 [arXiv: 1812.08293] [InSPIRE].

[21] H. Davoudiasl, I. Lewis and E. Ponton, Electroweak Phase Transition, Higgs Diphoton Rate, and New Heavy Fermions, Phys. Rev. D 87 (2013) 093001 [arXiv:1211.3449] [INSPIRE].

[22] O. Matsedonskyi and G. Servant, High-Temperature Electroweak Symmetry Non-Restoration from New Fermions and Implications for Baryogenesis, JHEP 09 (2020) 012 [arXiv:2002.05174] [INSPIRE].

[23] S.R. Coleman and E.J. Weinberg, Radiative Corrections as the Origin of Spontaneous Symmetry Breaking, Phys. Rev. D 7 (1973) 1888 [INSPIRE].

[24] M. Bando, T. Kugo, N. Maekawa and H. Nakano, Improving the effective potential, Phys. Lett. B 301 (1993) 83 [hep-ph/9210228] [INSPIRE].

[25] M. Bando, T. Kugo, N. Maekawa and H. Nakano, Improving the effective potential: Multimass scale case, Prog. Theor. Phys. 90 (1993) 405 [hep-ph/9210229] [INSPIRE].

[26] L. Chataignier, T. Prokopec, M.G. Schmidt and B. Swiezewska, Single-scale Renormalisation Group Improvement of Multi-scale Effective Potentials, JHEP 03 (2018) 014 [arXiv: 1801.05258] [INSPIRE].

[27] S. Weinberg, Effective Gauge Theories, Phys. Lett. B 91 (1980) 51 [InSPIRE].

[28] W. Buchmüller and D. Wyler, Effective Lagrangian Analysis of New Interactions and Flavor Conservation, Nucl. Phys. B 268 (1986) 621 [INSPIRE].

[29] M.K. Gaillard, The Effective One Loop Lagrangian With Derivative Couplings, Nucl. Phys. B 268 (1986) 669 [INSPIRE].

[30] L.-H. Chan, Derivative Expansion for the One Loop Effective Actions With Internal Symmetry, Phys. Rev. Lett. 57 (1986) 1199 [INSPIRE].

[31] O. Cheyette, Effective Action for the Standard Model With Large Higgs Mass, Nucl. Phys. B 297 (1988) 183 [INSPIRE].

[32] B. Henning, X. Lu and H. Murayama, How to use the Standard Model effective field theory, JHEP 01 (2016) 023 [arXiv:1412.1837] [INSPIRE].

[33] R. Huo, Standard Model Effective Field Theory: Integrating out Vector-Like Fermions, JHEP 09 (2015) 037 [arXiv: 1506.00840] [INSPIRE].

[34] A. Drozd, J. Ellis, J. Quevillon and T. You, The Universal One-Loop Effective Action, JHEP 03 (2016) 180 [arXiv:1512.03003] [InSPIRE].

[35] B. Henning, X. Lu and H. Murayama, One-loop Matching and Running with Covariant Derivative Expansion, JHEP 01 (2018) 123 [arXiv: 1604.01019] [INSPIRE].

[36] S.A.R. Ellis, J. Quevillon, T. You and Z. Zhang, Mixed heavy-light matching in the Universal One-Loop Effective Action, Phys. Lett. B 762 (2016) 166 [arXiv:1604.02445] [INSPIRE].

[37] J. Fuentes-Martin, J. Portoles and P. Ruiz-Femenia, Integrating out heavy particles with functional methods: a simplified framework, JHEP 09 (2016) 156 [arXiv:1607.02142] [INSPIRE]. 
[38] Z. Zhang, Covariant diagrams for one-loop matching, JHEP 05 (2017) 152 [arXiv: 1610.00710] [INSPIRE].

[39] S.A.R. Ellis, J. Quevillon, T. You and Z. Zhang, Extending the Universal One-Loop Effective Action: Heavy-Light Coefficients, JHEP 08 (2017) 054 [arXiv:1706. 07765] [INSPIRE].

[40] M. Krämer, B. Summ and A. Voigt, Completing the scalar and fermionic Universal One-Loop Effective Action, JHEP 01 (2020) 079 [arXiv: 1908.04798] [INSPIRE].

[41] T. Cohen, M. Freytsis and X. Lu, Functional Methods for Heavy Quark Effective Theory, JHEP 06 (2020) 164 [arXiv:1912.08814] [INSPIRE].

[42] S.A.R. Ellis, J. Quevillon, P.N.H. Vuong, T. You and Z. Zhang, The Fermionic Universal One-Loop Effective Action, JHEP 11 (2020) 078 [arXiv:2006.16260] [INSPIRE].

[43] A. Angelescu and P. Huang, Integrating Out New Fermions at One Loop, JHEP 01 (2021) 049 [arXiv: 2006.16532] [INSPIRE].

[44] I. Masina, G. Nardini and M. Quirós, Electroweak vacuum stability and finite quadratic radiative corrections, Phys. Rev. D 92 (2015) 035003 [arXiv:1502.06525] [INSPIRE].

[45] R. Jackiw, Functional evaluation of the effective potential, Phys. Rev. D 9 (1974) 1686 [INSPIRE].

[46] J. Fleischer and F. Jegerlehner, Radiative Corrections to Higgs Decays in the Extended Weinberg-Salam Model, Phys. Rev. D 23 (1981) 2001 [INSPIRE].

[47] A. Denner, Techniques for calculation of electroweak radiative corrections at the one loop level and results for W physics at LEP-200, Fortsch. Phys. 41 (1993) 307 [arXiv:0709.1075] [INSPIRE].

[48] W. Loinaz and R.S. Willey, Gauge dependence of lower bounds on the Higgs mass derived from electroweak vacuum stability constraints, Phys. Rev. D 56 (1997) 7416 [hep-ph/9702321] [INSPIRE].

[49] S. Kanemura, Y. Okada, E. Senaha and C.P. Yuan, Higgs coupling constants as a probe of new physics, Phys. Rev. D 70 (2004) 115002 [hep-ph/0408364] [INSPIRE].

[50] S. Actis, A. Ferroglia, M. Passera and G. Passarino, Two-Loop Renormalization in the Standard Model. Part I: Prolegomena, Nucl. Phys. B 777 (2007) 1 [hep-ph/0612122] [INSPIRE].

[51] A. Denner, L. Jenniches, J.-N. Lang and C. Sturm, Gauge-independent $\overline{M S}$ renormalization in the 2HDM, JHEP 09 (2016) 115 [arXiv:1607.07352] [INSPIRE].

[52] A. Denner and S. Dittmaier, Electroweak Radiative Corrections for Collider Physics, Phys. Rept. 864 (2020) 1 [arXiv: 1912.06823] [InSPIRE].

[53] Qing-Hong Cao, Katsuya Hashino, Xuxiang Li, Zhe Ren and Jiang-Hao Yu, in preparation.

[54] M. Chala, C. Krause and G. Nardini, Signals of the electroweak phase transition at colliders and gravitational wave observatories, JHEP 07 (2018) 062 [arXiv: 1802.02168] [INSPIRE].

[55] M. Postma and G. White, Cosmological phase transitions: is effective field theory just a toy?, JHEP 03 (2021) 280 [arXiv:2012.03953] [INSPIRE].

[56] D. Croon, O. Gould, P. Schicho, T.V.I. Tenkanen and G. White, Theoretical uncertainties for cosmological first-order phase transitions, JHEP 04 (2021) 055 [arXiv:2009.10080] [INSPIRE]. 
[57] P.M. Schicho, T.V.I. Tenkanen and J. Österman, Robust approach to thermal resummation: Standard Model meets a singlet, JHEP 06 (2021) 130 [arXiv:2102.11145] [INSPIRE].

[58] S. Bhattacharya, N. Sahoo and N. Sahu, Minimal vectorlike leptonic dark matter and signatures at the LHC, Phys. Rev. D 93 (2016) 115040 [arXiv:1510.02760] [INSPIRE].

[59] CMS collaboration, Search for vector-like leptons in multilepton final states in proton-proton collisions at $\sqrt{s}=13 \mathrm{TeV}$, Phys. Rev. D 100 (2019) 052003 [arXiv: 1905.10853] [InSPIRE].

[60] Particle Data Group collaboration, Review of Particle Physics, PTEP 2020 (2020) 083C01 [INSPIRE].

[61] ATLAS collaboration, Constraints on the Higgs boson self-coupling from the combination of single-Higgs and double-Higgs production analyses performed with the ATLAS experiment, Tech. Rep. ATL-PHYS-PROC-2020-114, CERN, Geneva (Dec, 2020), DOI.

[62] K. Fujii et al., Physics Case for the International Linear Collider, arXiv:1506.05992 [INSPIRE].

[63] D. Gonçalves, T. Han, F. Kling, T. Plehn and M. Takeuchi, Higgs boson pair production at future hadron colliders: From kinematics to dynamics, Phys. Rev. D 97 (2018) 113004 [arXiv: 1802.04319] [INSPIRE].

[64] J. Chang, K. Cheung, J.S. Lee, C.-T. Lu and J. Park, Higgs-boson-pair production $H\left(\rightarrow b b^{-}\right) H(\rightarrow \gamma \gamma)$ from gluon fusion at the HL-LHC and HL-100 TeV hadron collider, Phys. Rev. D 100 (2019) 096001 [arXiv: 1804.07130] [INSPIRE].

[65] CLICDP collaboration, Double Higgs boson production and Higgs self-coupling extraction at CLIC, Eur. Phys. J. C 80 (2020) 1010 [arXiv:1901.05897] [InSPIRE].

[66] M. Cepeda et al., Report from Working Group 2: Higgs Physics at the HL-LHC and HE-LHC, CERN Yellow Rep. Monogr. 7 (2019) 221 [arXiv: 1902.00134] [InSPIRE].

[67] S. Gopalakrishna and A. Velusamy, Higgs vacuum stability with vectorlike fermions, Phys. Rev. D 99 (2019) 115020 [arXiv: 1812.11303] [INSPIRE]. 